\title{
OPEN PROBLEMS IN SYMBOLIC DYNAMICS
}

\author{
MIKE BOYLE
}

\section{Contents}

1. Introduction 2

2. Definitions 3

3. Classification of SFTs and the Little Shift Equivalence Conjecture 4

4. Range of the dimension representation 6

5. Positive Rational Shift Equivalence Conjecture $\quad 7$

6. The Generalized Spectral Conjecture 7

7. The Equal Entropy Factors Conjecture for SFTs 8

8. The factors problem for sofic shifts 9

9. The Good Finitary Conjecture for Markov shifts 10

10. What can be a beta function? 11

11. Structure of expansive subdynamics 12

12. Can nonSFT expansive maps commute with irreducible SFTs? 13

13. The Commuting Powers Conjecture for SFTs 14

14. Jointly invariant measures 15

15. $\mathbb{Z}^{d}$ SFTs 16

16. Stable limit sets of cellular automata 17

$\begin{array}{ll}\text { 17. Algebraic } \mathbb{Z}^{d} \text { SFTs } & 18\end{array}$

18. Finitary codes and Markov random fields 18

19. Decidability problems 20

20. Onesided SFTs: the embedding problem 21

21. Onesided sofic shifts: the classification problem 21

22. The Virtual FOG Conjecture for automorphisms of a mixing SFT 23

23. Topological orbit equivalence 24

24. Symbolic extension entropy and entropy structure 25

25. Cellular automata and periodic points 26

26. Cellular automata on big groups 27

27. Entropy conjugacy and countable state Markov chains 27

28. Beta shifts: Salem numbers and intrinsic ergodicity 28

29. Adler's Renewal Question 28

30. The Road Coloring Problem 28

31. Parry's Finiteness Question for skew products 29

32. Classification of sofic shifts 29

33. Classification and flow equivalence of general shifts 31

34. The Pisot Conjecture 33

35. Nivat's Conjecture 34

Date: March 19, 2008.

2000 Mathematics Subject Classification. 37B10. 
Appendix A. Commuting SFTs and periodic points

Appendix B. Commuting SFTs from commuting matrices, following Nasu 36 Appendix C. LR Textile Systems 37

Appendix D. Commuting SFTs from matrices commuting on dimension 39

Appendix E. Primitive matrices SE but not SSE over $\mathbb{Z}_{+}[1 / p] \quad 40$

Appendix F. Examples for the onesided sofic classification problem 41

References

\section{INTRODUCTION}

More than most topics, symbolic dynamics is defined by its problems. Many of the most natural problems (e.g., when do two matrices define topologically conjugate shifts of finite type) have been open for a long time. Many of the problems in this survey are, so far as I can tell, very difficult; on the other hand, this is a subject in which a clever idea or example will sometimes make a longstanding problem look easy.

With some exceptions, I've tried to focus on sharp problems rather than the broader investigations they represent. I'll be satisfied if any mathematician is spurred by this survey to solve any of the problems it contains. I'm also thinking of this survey as a snapshot from today.

The price of including many problems has been that in many cases the presentation is quite condensed. However, I've also included two appendices which give a little exposition of the underappreciated "textile systems" theory of Nasu. The other appendices contain previously unpublished results relevant to some of the open problems.

This survey is concerned overwhelming with "internal" questions of symbolic dynamics (whatever it is), rather than its applications. For example, there is nothing about the use of symbolic dynamics in geometry (see [99] for a survey of symbolic representations of geodesics on surfaces of constant negative curvature) or in applications to specific nonsymbolic systems. Another omission is any problem on automatic sequences; here, consult the excellent $[3,189]$ for background and many open problems.

The bibliography is huge, but it (and the paper) would be much longer if there were an attempt to record all the significant contributions to the problems considered. I've tried to include enough references that someone interested in a problem will find the appropriate papers and contributions, and apologize to those neglected.

The survey naturally reflects my own biases and interests, and I'm grateful to the experts whose input has ameliorated that tendency, however modestly. For feedback, suggestions and corrections, I especially thank Valerie Berthe, Jerome Buzzi, Fabien Durand, Bruce Kitchens, Alejandro Maass, Kengo Matsumoto, Masakazu Nasu, Marcus Pivato and Jeff Steif. I'm also grateful to Larry Washington for help with the number theory in Appendix E. Most of this paper was written as a guest of Universidad de Chile in Santiago; I was very fortunate to work in such a friendly, beautiful and stimulating environment.

This paper is dedicated to Misha Brin, on the occasion of his 60th birthday, with thanks and admiration, for so many years of brilliance, wit, generosity, leadership and friendship. 


\section{Definitions}

We assume some familiarity with basic symbolic dynamics. Good introductions can be found in the standard texts $[127,119]$ (also see $[16,126]$ ). Still, we give some background and definitions now.

Let $A$ be an $n \times n$ matrix with entries from the nonnegative integers $\mathbb{Z}_{+}=\mathbb{N} \cup\{0\}$. Let $\mathfrak{G}_{A}$ be a directed graph with vertices $1,2, \ldots, n$ such that $A(i, j)$ is the number of edges from vertex $i$ to vertex $j$. Let $\mathcal{E}$ be the edge set of $\mathfrak{G}_{A}$. Let $\mathcal{E}^{\mathbb{Z}}$ have the product topology of the discrete topology. The shift map $\sigma: \mathcal{E}^{\mathbb{Z}} \rightarrow \mathcal{E}^{\mathbb{Z}}$ is defined by $(\sigma x)(n)=x(n+1)$. This is the full shift on alphabet $\mathcal{E}$ (it is a version of the full shift on $|\mathcal{E}|$ symbols). Let $\Sigma_{A}$ be the set of $x$ in $\mathcal{E}^{\mathbb{Z}}$ corresponding to legal walks through $\mathfrak{G}_{A}$ (i.e., for all $n$ the terminal vertex of $x(n)$ equals the initial vertex of $x(n+1))$. Then $\Sigma_{A}$ is compact metrizable and shift invariant. The restriction $\sigma_{A}$ of $\sigma$ to $\Sigma_{A}$ is an edge shift of finite type (edge SFT). (Often $\Sigma_{A}$ is called an SFT, or the same symbol is used for both the map and the space. Context clarifies this.) An SFT is any topological dynamical system which is topologically conjugate (isomorphic) to an edge SFT. The restriction of the shift to a closed invariant subset $X$ of a full shift $Y$ is SFT if and only if there is a finite set $\mathcal{W}$ such that $X=\left\{x \in Y: \forall i, j x_{i} \cdots x_{j} \notin \mathcal{W}\right\}$.

A onesided SFT is defined in just the same fashion, but with the use of $\mathcal{E}^{\mathbb{Z}_{+}}$ (onesided sequences) in place of $\mathcal{E}^{\mathbb{Z}}$. A onesided SFT is a continuous open map, but is rarely injective.

We may also begin with a finite set $E$ (no longer edges) and the compact metrizable space $E^{\mathbb{Z}^{d}}$. For $d \geq 1$, the $\mathbb{Z}^{d}$ shift action on $E^{\mathbb{Z}^{d}}$ is defined for $\mathbf{m}, \mathbf{n}$ in $\mathbb{Z}^{d}$ by $\left(\sigma^{\mathbf{n}} x\right)(\mathbf{m})=x(\mathbf{m}+\mathbf{n})$. A $\mathbb{Z}^{d}$ SFT $X$ is defined for $d>1$ as it was for $d=1$; the domain is the set of all $x$ in $E^{\mathbb{Z}^{d}}$ which avoid some finite set of finite configurations. (That is, there are finite subsets $S_{1}, \ldots, S_{k}$ of $\mathbb{Z}^{d}$, and functions $f_{i}: S_{i} \rightarrow E$, such that $X$ is the set of all $x \in E^{\mathbb{Z}^{d}}$ such that $\forall \mathbf{n} \in \mathbb{Z}^{d}$, the restriction of $x$ to $\mathbf{n}+S_{i}$ does not equal $f_{i}$.) If we write just SFT, we will mean a $\mathbb{Z}$ SFT.

A sofic shift is a subshift which is the image of an SFT under a block code; i.e. it is a subshift which is a quotient, or factor, of an SFT. A $\mathbb{Z}^{d}$ sofic shift is a similarly a $\mathbb{Z}^{d}$ subshift which is a quotient of a $\mathbb{Z}^{d}$ SFT.

Let $\mathcal{R}$ be a semiring containing 0 and 1 (additive and multipicative identities). Matrices $A, B$ over $\mathcal{R}$ (i.e. with entries in $\mathcal{R}$ ) are elementary strong shift equivalent over $\mathcal{R}$ (ESSE- $\mathcal{R})$ if there exist matrices $U, V$ over $\mathcal{R}$ such that $A=U V$ and $B=V U$. They are strong shift equivalent over $\mathcal{R}(\mathrm{SSE}-\mathcal{R})$ if there is an $\ell \in \mathbb{N}$ and matrices $A=A_{0}, A_{1}, \ldots, A_{\ell}=B$ such that $A_{i}$ and $A_{i+1}$ are (ESSE- $\mathcal{R}$ ) for $0 \leq i<\ell$. Here $\ell$ is called the $\operatorname{lag}$ of the given strong shift equivalence. $A$ and $B$ are shift equivalent over $\mathcal{R}(\mathrm{SE}-\mathcal{R})$ if there exist matrices $U, V$ and a positive integer $\ell$ such that the following equations hold:

$$
A^{\ell}=U V, \quad B^{\ell}=V U, \quad A U=U B, \quad V A=B V .
$$

The integer $\ell$ is called the lag of the given shift equivalence.

Square matrices $A, B$ over $\mathbb{Z}_{+}$define topologically conjugate edge SFTs if and only if $A$ and $B$ are SSE- $\mathbb{Z}_{+}$; also $A^{k}, B^{k}$ are SSE- $\mathbb{Z}_{+}$for all but finitely many $k \in \mathbb{N}$ if and only if $A$ and $B$ are SE- $\mathbb{Z}_{+}$.

Let $A$ be an $n \times n$ matrix with integer entries, acting on row vectors. Define $V_{A}$ as the image of $\mathbb{Q}^{n}$ under $A^{n}$ and $G_{A}=\left\{v \in V_{A}: \exists k>0, v A^{k} \in \mathbb{Z}^{n}\right\}$. The group $G_{A}$ is a concrete version of the direct limit group $\lim _{A} \mathbb{Z}^{n}$, convenient for 
our purposes. We will call $G_{A}$ a dimension group, although we will not need to consider the natural order structure which makes it a (stationary) dimension group. The dimension module $\mathcal{G}_{A}$ of the matrix $A$ (or SFT $\sigma_{A}$ ) is the group $G_{A}$ together with its group automorphism $\widehat{A}$ defined by $v \mapsto v A$. For notation below, let $\widehat{A}$ be identified with a matrix which acts like $A$ on $V_{A}$ and annihilates $\operatorname{ker}\left(A^{n}\right)$; and identify $(\widehat{A})^{-1}$ with a matrix which is the inverse isomorphism on $V_{A}$ and also annihilates $\operatorname{ker}\left(A^{n}\right)$.

Suppose $f: X \rightarrow X$ is a continuous map. An automorphism of $f$ is a homeomorphism commuting with $f$, and Aut $(f)$ is the automorphism group of $f$. If $\phi$ is an automorphism of an SFT $\sigma_{A}$, then $\phi$ acts on the dimension module $\mathcal{G}_{A}$ (that is, it defines a group isomorphism $\widehat{\phi}: G_{A} \rightarrow G_{A}$ which commutes with $\widehat{A}$ ). This may be defined in terms of the action of $\phi$ on certain subsets of $\Sigma_{A}$, following Krieger [43]. Here we will give the Wagoner definition in terms of matrices [190].

Let $A=U V, B=V U$ be an elementary strong shift equivalence over $\mathbb{Z}_{+}$. We view the vertex sets of the directed graphs $\mathfrak{G}_{A} \mathfrak{G}_{B}$ as disjoint. We view $U$ as the adjacency matrix for a set of edges from $\mathfrak{G}_{A}$ vertices to $\mathfrak{G}_{B}$ vertices, and similarly $V$ for edges from $\mathfrak{G}_{A}$. Say $u v$ is a $U, V$ path from $i$ to $j$ if, for some $k, u$ is a $U$ edge from $i$ to $k$ and $v$ is a $V$ edge from $k$ to $j$. Now the number of edges in $\mathcal{E}_{A}$ from vertex $i$ to vertex $j$ equals the number of $U, V$ paths from $i$ to $j$; and a similar statement holds for $B, V, U$. Thus we can rename elements of $\mathcal{E}_{A}$ and $\mathcal{E}_{B}$ as $U, V$ and $V, U$ paths.

Now a point $x$ in $\Sigma_{A}$ is

$$
x=\ldots x_{-1} x_{0} x_{1} \ldots=\ldots\left(u_{-1} v_{-1}\right)\left(u_{0} v_{0}\right)\left(u_{1} v_{1}\right) \ldots .
$$

The ESSE of forward type defined by the pair $(U, V)$ is simply the map which sends this $x$ to the point

$$
y=\ldots y_{-1} y_{0} y_{1} \ldots=\ldots\left(v_{-1} u_{0}\right)\left(v_{0} u_{1}\right)\left(v_{1} u_{2}\right) \ldots .
$$

A forward conjugacy is a composition of ESSE's of forward type. Any topological conjugacy $\phi$ from an SFT $\sigma_{A}$ to an SFT $\sigma_{B}$ is a composition of ESSEs of forward type, given say by $\left(U_{1}, V_{1}\right),\left(U_{2}, V_{2}\right), \ldots\left(U_{\ell}, V_{\ell}\right)$, followed by some (possibly negative) power $k$ of the shift. The induced isomorphism $G_{A} \rightarrow G_{B}$ is then defined by $v \mapsto v U_{1} U_{2} \cdots U_{\ell} \widehat{B}^{k}$ (the isomorphism depends on $\phi$, not on the particular chain of matrices used to implement $\phi$ ). When $A=B$, this isomorphism is $\widehat{\phi}$. The map from $\operatorname{Aut}\left(\sigma_{A}\right)$ defined by $\phi \rightarrow \widehat{\phi}$ is the dimension representation.

\section{Classification of SFTs and the Little Shift Equivalence Conjecture}

Conjecture 3.1 ("Little shift equivalence conjecture"). Suppose A is a nonnegative matrix over $\mathbb{Z}$ which has a unique and simple nonzero eigenvalue $n$. Then $A$ is strong shift equivalent over $\mathbb{Z}_{+}$to $[n]$.

Conjecture 3.1 is a concrete instance of the following central problem of symbolic dynamics.

Problem 3.2. Classify SFTs up to topological conjugacy. In particular, give a procedure which decides when $A$ and $B$ define topologically conjugate SFTs. 
Of course, one could take the view that providing the requested decision procedure is the same as providing a classification; on the other hand, one could hope for some additional conceptual structure.

At present we have no conjectural solution to Problem 3.2. For many years, the best hope was Williams' Conjecture [192]: if $A$ and $B$ are shift equivalent over $\mathbb{Z}_{+}$, then they are strong shift equivalent over $\mathbb{Z}_{+}$. (So in general, we now say a Williams problem is to determine in some class whether shift equivalence implies strong shift equivalence [21].) The conjecture was refuted by Kim and Roush, first in the reducible case [109] and then in the primitive case [112]. Their arguments took place within an algebraic-topology classification structure developed over years by Wagoner; see [190] and its references for this very interesting story and further approaches to the classification problem. We make some remarks.

(1) If $\mathbb{Z}_{+}$in Conjecture 3.1 is replaced by $\mathbb{Q}_{+}$, then by work of Kim and Roush [103] the conjecture is known to be true (see Section 5).

(2) The Kim-Roush argument establishing two matrices $A, B$ as counterexamples to Williams' Conjecture appeals in particular to zero/+ patterns in traces of powers of the matrices $A, B$. The traces of all powers of matrices involved in 3.1 are positive.

(3) It is very easy to classify irreducible SFTs given a classification for mixing SFTs. So, Problem 3.2 naturally splits into the mixing case (where the Wagoner setting used in [112] applies, because primitive matrices over $\mathbb{Z}$ are $\mathrm{SSE}-\mathbb{Z}$ iff they are $\mathrm{SE}-\mathbb{Z}_{+}$) and the reducible case (for this see Section $4)$.

(4) The idea of strong shift equivalence allows a unified approach to several classification problems in symbolic dynamics, including Problem 3.2. There is another general approach to these problems, the "positive K-theory" $[23,49]$. This approach may be relevant to the solution of Problem 3.2.

Conjecture 3.1 is a special case of Williams' Conjecture. Currently, we essentially have no general sufficient conditions for existence of a conjugacy between given $\sigma_{A}$ and $\sigma_{B}$.

By special methods, Williams' Conjecture is known to be true if $A$ and $B$ are both $2 \times 2$ AND have determinant $\geq-1[9,62,192]$. Kirby Baker has more to say about some small matrices [10].

Here are a few specific open instances of Williams' problem.

(1) Suppose $A$ and $B$ are $2 \times 2$ and SE over $\mathbb{Z}_{+}$with determinant less than -1 . Must $A$ and $B$ be SSE over $\mathbb{Z}_{+}$?

(2) Let $A$ be "Ashley's eight-by-eight", the $8 \times 8$ matrix which is the sum of the permutation matrices for the permutations which in cycle notation are (12345678) and (1)(2)(374865). Then $A$ is SE- $\mathbb{Z}_{+}$to [2]. Is $A$ SSE- $\mathbb{Z}_{+}$to [2]?

Up to symmetries of $A$, this question is [119, Example 2.2.7] and it is due to Jon Ashley in 1989 [119, Chapter notes, p.60]. For any matrix $B$ SE over $\mathbb{Z}_{+}$to [2], there is $k \in \mathbb{N}$ such that $B$ is SSE over $\mathbb{Z}_{+}$to a matrix of size $2^{k}$ with all row sums and all column sums equal to [2] [134]. Except for $A$ (and some matrices SSE over $\mathbb{Z}_{+}$to $A$ by natural symmetries), all such matrices $B$ of size $2^{k}$ with $k \leq 3$ were checked by Ashley and found to be SSE- $\mathbb{Z}_{+}$to the $1 \times 1$ matrix [2] [Jon Ashley, personal communication]. 
(3) Given $k \geq 2 \in \mathbb{N}$, define the matrices

$$
A_{k}=\left(\begin{array}{cc}
1 & k \\
k-1 & 1
\end{array}\right) \quad \text { and } \quad B_{k}=\left(\begin{array}{cc}
1 & k(k-1) \\
1 & 1
\end{array}\right)
$$

Then $A_{k}$ is SE- $\mathbb{Z}+$ to $B_{k}$, but for $k \geq 4$ it is not known if $A_{k}$ is SE- $\mathbb{Z}+$ to $B_{k}[127$, Example 7.3.13]

For more on strong shift equivalence see $[86,127,21,190]$ and their references.

\section{RANGE OF THE DIMENSION REPRESENTATION}

Let $\sigma_{A}$ be a mixing SFT, defined by a primitive matrix $A$ over $\mathbb{Z}_{+}$, with dimension module $\mathcal{G}_{A}$. Let $\operatorname{Aut}_{+}\left(\mathcal{G}_{A}\right)$ denote the group of positive automorphisms of the dimension module $\mathcal{G}_{A}$; these are the module automorphisms of $\mathcal{G}_{A}$ which multiply the Perron eigendirection of $A$ by a positive number.

For example, with $A=[18]$, as a group $\mathcal{G}_{A}$ is isomorphic to $\mathbb{Z}[1 / 18]$; all isomorphisms from this group to itself commute with the action of $A$; these isomorphisms are multiplication by a number of the form $\pm 2^{i} 3^{j}$ with $(i, j) \in \mathbb{Z}^{2}$; and finally for the positivity condition we allow only $2^{i} 3^{j}$, so that in this case the group $\operatorname{Aut}_{+}\left(\mathcal{G}_{A}\right)$ is isomorphic to $\mathbb{Z}^{2}$. For $A=\left(\begin{array}{ll}1 & 1 \\ 1 & 0\end{array}\right)$, as a group $\mathcal{G}_{A}$ is isomorphic to $\mathbb{Z}^{2}$, but the only elements of $\mathrm{GL}(2, \mathbb{Z})$ commuting with $A$ have the form $\pm A^{n}, n \in \mathbb{Z}$, and therefore $\operatorname{Aut}_{+}\left(\mathcal{G}_{A}\right)$ is isomorphic to $\mathbb{Z}$.

If $\phi$ is an automorphism of $\sigma_{A}$, then $\phi$ induces a positive isomorphism $\widehat{\phi}: \mathcal{G}_{A} \rightarrow$ $\mathcal{G}_{A}$. The map

$$
\begin{aligned}
\rho_{A}: \operatorname{Aut}\left(\sigma_{A}\right) & \rightarrow \operatorname{Aut}_{+}\left(\mathcal{G}_{A}\right) \\
\phi & \mapsto \widehat{\phi}
\end{aligned}
$$

is the dimension representation of the automorphism group of $S_{A}$. An automorphism is inert if it is in the kernel of the dimension representation.

Problem 4.1. Given a mixing SFT $S_{A}$, determine the range of its dimension representation.

In general the dimension representation is not surjective [114]. The known obstruction involves the sign-gyration-compatibility-homomorphism SGGC, which is a homomorphism from $\operatorname{Aut}\left(\sigma_{A}\right)$ which for each $n$ assigns to an element $U$ of $\operatorname{Aut}\left(\sigma_{A}\right)$ an element $\operatorname{SGCC}_{n}(U)$ of $\mathbb{Z} / n$ which is computed from the action of $U$ on points of periods $n, n / 2, n / 4, \ldots$. The Factorization Theorem of Kim, Roush and Wagoner shows that SGCC is determined by and computable from the dimension representation $[114,112]$. It can happen for a specific element $r$ of $\operatorname{Aut}_{+}\left(\mathcal{G}_{A}\right)$ and $n \in \mathbb{N}$ that $r$ can be in the range of $\rho_{A}$ only if $\mathrm{SGCC}_{n}$ is nontrivial, while $\sigma_{A}$ has no points of period $n, n / 2, n / 4, \ldots$ [114]. In this case $r$ cannot be in the range of $\rho_{A}$. It is not known whether this type of SGCC obstruction is the only obstruction to realizing an element in the range of $\rho_{A}$.

We note two significant applications for a solution of Problem 4.1.

(1) (Classification of shifts of finite type up to topological conjugacy.) Given a classification for irreducible SFTs, the classfication of general (possibly reducible) SFTs requires the solution of Problem 4.1, and can be reduced to a solution of Problem $4.1[109,108]$. 
(2) (Solution of the extension problem.) The extension problem is to characterize which automorphisms of subsystems of a mixing SFT $\sigma_{A}$ extend to automorphisms of $\sigma_{A}$. Following $[115,116]$, we know exactly which automorphisms of finite (or more generally proper [39]) subsystems of a mixing SFT $\sigma_{A}$ extend to automorphisms of $\sigma_{A}$ which act trivially on the dimension group of $\sigma_{A}$. By the Factorization Theorem, the extension problem then reduces (at least in the typical case that $\operatorname{Aut}_{+}\left(\mathcal{G}_{A}\right)$ is finitely generated [43]) to knowing the range of the dimension representation.

Following earlier constructions [43, 29, 39, 148], Kim, Roush and Wagoner developed powerful (but difficult) new "positive K-theory" methods for constructing automorphisms $[115,116,110]$, which may be relevant to solving Problem 4.1.

\section{Positive Rational Shift Equivalence Conjecture}

Conjecture 5.1 (Positive Rational Shift Equivalence Conjecture). Suppose A,B are square positive matrices which are shift equivalent over $\mathbb{Q}_{+}$Then $A, B$ are strong shift equivalent over $\mathbb{Q}_{+}$.

Kim and Roush ([103], following [107, 106, 105, 101] proved Conjecture 5.1 under the assumption that $A$ (and thus also $B$ ) has a single nonzero eigenvalue, $n$. There is no other result known which gives a classification up to SSE over $\mathbb{Q}_{+}$of all primitive matrices in the $\mathrm{SE}-\mathbb{Q}_{+}$class of a primitive matrix over $\mathbb{Q}$. The corresponding statement for $\mathbb{Z}$ is the "Little Shift Equivalence Conjecture" 3.1 .

The approach Kim and Roush developed for their argument analyzes paths of matrix similarities over $\mathbb{R}$, associated covers of the paths by neighborhoods yielding an elementary similarity, and the use of these and compactness (the covers have finite subcovers) to find chains of elementary SSE's over $\mathbb{Q}_{+}$. This framework is rather general and may be very relevant to Conjecture 5.1.

The Rational Shift Equivalence Conjecture is obtained by deleting the word "positive" from Conjecture 5.1; it is also open. The positive case seems to be the correct first step. The great advantage of positive matrices, exploited by Kim and Roush, is that they are stable under perturbation.

A primitive matrix over $\mathbb{Q}_{+}$with positive trace is SSE over $\mathbb{Z}_{+}$to a positive matrix; this is a special case of a much stronger result of Kim and Roush [101, Theorem 6.5]. Thus Conjecture 5.1 is really a conjecture for primitive matrices over $\mathbb{Q}_{+}$with positive trace, which is a major special case for the general problem of deciding when matrices are strong shift equivalent over $\mathbb{Q}_{+}$.

It is natural to ask whether Conjecture 5.1 might be true if $\mathbb{Q}$ were replaced by some other nondiscrete subring of $\mathbb{R}$. In Appendix E, we will see that the Rational Shift Equivalence Conjecture would be false if in its statement $\mathbb{Q}$ were replaced by $\mathbb{Z}[1 / p]$ with $p$ a rational prime. However, we have no example of a unital subring $\mathbb{S}$ of $\mathbb{R}$ and two positive matrices which are shift equivalent over $\mathbb{S}_{+}$and which are not strong equivalent over $\mathbb{S}_{+}$.

\section{The Generalized Spectral Conjecture}

For SFTs, in addition to knowing when they are topologically conjugate, we would like to understand the class of matrices which occur as their defining nonnegative integral matrices. The fundamental problem here (as explained in [33]) is to understand what matrices are primitive integral matrices. This is addressed by 
the $\mathbb{S}=\mathbb{Z}$ cases of the next two conjectures of myself and David Handelman. We say that a $k$-tuple $\Lambda$ of complex numbers is the nonzero spectrum of a matrix $A$ if for some $j \geq 0$ its characteristic polynomial $\chi_{A}(t)$ equals $t^{j}\left(t-\lambda_{1}\right) \cdots\left(t-\lambda_{k}\right)$. For $n \in \mathbb{N}$, we define

$$
\begin{aligned}
\operatorname{tr}\left(\Lambda^{n}\right) & =\sum_{i=1}^{k}\left(\lambda_{i}\right)^{n} \\
\operatorname{tr}_{n}(\Lambda) & =\sum_{j: j \mid n} \mu\left(\frac{n}{j}\right) \operatorname{tr}\left(\Lambda^{j}\right)
\end{aligned}
$$

where $\mu$ is the Mobius function. For example,

$$
\operatorname{tr}_{12}(\Lambda)=\operatorname{tr}\left(\Lambda^{12}\right)-\operatorname{tr}\left(\Lambda^{6}\right)-\operatorname{tr}\left(\Lambda^{4}\right)+\operatorname{tr}\left(\Lambda^{2}\right) .
$$

Conjecture 6.1 (Spectral Conjecture). Let $\mathbb{S}$ be a unital subring of $\mathbb{R}$ and let $\Lambda=\left(\lambda_{1}, \ldots, \lambda_{k}\right)$ be a $k$-tuple of complex numbers. Then $\Lambda$ is the nonzero spectrum of a primitive matrix over $\mathbb{S}$ if and only if the following necessary conditions hold.

(1) (Perron condition) There exists $i$ such that $\lambda_{i}$ is a positive real number and $\lambda_{i}>\left|\lambda_{j}\right|$ if $j \neq i$ and $1 \leq j \leq k$.

(2) (Galois condition) The polynomial $\left(t-\lambda_{1}\right) \cdots\left(t-\lambda_{k}\right)$ has all coefficients in $\mathbb{S}$.

(3) (Trace condition) If $\mathbb{S} \neq \mathbb{Z}$, then the following hold for all $n \in \mathbb{N}$ :

- $\operatorname{tr}\left(\Lambda^{n}\right) \geq 0$

- if $j$ divides $n$ and $\operatorname{tr}\left(\Lambda^{j}\right)>0$ then $\operatorname{tr}\left(\Lambda^{n}\right)>0$.

If $\mathbb{S}=\mathbb{Z}$, then $\operatorname{tr}_{n}(\Lambda) \geq 0$, for all $n \in \mathbb{N}$.

Strong shift equivalence is an appropriate stable version of similarity (see [23], following [133]); from this viewpoint, the next conjecture is a realization conjecture for the stable algebraic structure of primitive matrices.

Conjecture 6.2 (Generalized Spectral Conjecture [33, 21]). Suppose $\mathbb{S}$ is a unital subring of $\mathbb{R}, A$ is a square matrix with entries in $\mathbb{S}$ and the nonzero spectrum of $A$ satisfies the conditions of the Spectral Conjecture. Then A is strong shift equivalent over $\mathbb{S}$ to a primitive matrix.

The Spectral Conjecture was proved for $\mathbb{S}=\mathbb{R}$ and various other cases in [33], and by completely different methods in $[113]$ for the key case $\mathbb{S}=\mathbb{Z}$. (From the $\mathbb{Z}$ result, we understand completely the possible zeta functions of mixing, irreducible and general shifts of finite type.) It should be possible to adapt the (very complicated) argument of $[113]$ to the case $\mathbb{S} \neq \mathbb{Z}$. The Generalized Spectral Conjecture was proved for $\mathbb{S}=\mathbb{Z}$ (and thus $\mathbb{Q}$ ) in [34] for the special case that all elements of the nonzero spectrum are rational. There is a related result with different methods in $[42]$.

\section{The Equal Entropy Factors Conjecture for SFTs}

A subshift $T$ is a factor or quotient of a subshift $S$ if there is a continuous surjection $\phi$ (a factor map) between their domains such that $\phi S=T \phi$. One of the basic questions about SFTs is when one is a factor of another. For SFTs, the main case of interest is the case in which the SFTs under consideration are irreducible.

If $T$ is a factor of $S$, then $h(T) \leq h(S)$. Given mixing SFTs $S, T$ with $h(S)>$ $h(T)$, the shift $T$ is a factor of $S$ if and only if the trivially necessary periodic point 
condition (item (2) in the conjecture below) holds [20]. The case $h(S)=h(T)$ is addressed by the following conjecture.

Conjecture 7.1. [21] Suppose $A$ and $B$ are irreducible matrices over $\mathbb{Z}$ with the same spectral radius $\lambda$ and the following hold:

(1) For all $n \in \mathbb{N}$, if $\operatorname{tr} A^{n}>0$ then $\operatorname{tr} B^{n}>0$.

(2) The dimension module of $B$ is a quotient of a closed submodule of the dimension module of $A$.

Then the SFT $\sigma_{B}$ is a quotient of the SFT $\sigma_{A}$.

The conditions (1) and (2) of the conjecture are necessary for the existence of a factor map $S_{A} \rightarrow S_{B}$ [121]. If condition (2) is strengthened to the condition that $\mathcal{G}_{B}$ is a quotient (resp. closed submodule) of $\mathcal{G}_{A}$, then the desired factor map does exist and it can be chosen to be right (resp. left) closing [5]. (The closing factor maps are the factor maps which are topologically equivalent to resolving maps.) If the conclusion is weakened to the statement that for all but finitely many $n,\left(S_{B}\right)^{n}$ is a factor of $\left(S_{A}\right)^{n}$, then again the conjecture is true (e.g. [45]), even without condition (1). It is easy to check that Conjecture 7.1 is true in general if and only if it is true with "irreducible" replaced by "primitive" (i.e., the SFTs are mixing).

The known general constructions of factor maps between equal entropy mixing SFTs are constructions of maps which are left or right closing (i.e. maps topologically conjugate to left or right resolving maps), and we understand a good deal about resolving and closing factor maps [2, 5, 6, 45]. However, an example of [121] indicates that there are almost certainly pairs $A, B$ which satisfy the assumptions of Conjecture 7.1 but for which no factor map from $\sigma_{A}$ to $\sigma_{B}$ can be a composition of closing maps; and an example of Kitchens [121] shows that not all factor maps between irreducible SFTs are compositions of closing maps. While there are some results which relate general factor maps between equal entropy irreducible SFTs to resolving factor maps [121, 22, 25], we still lack the general constructions of nonclosing maps needed to prove Conjecture 7.1.

At this time, so far as I know, nobody has worked out a reduction of the general factors problem for equal entropy SFTs to the factors problem for irreducible SFTs. However, it appears from [12] that this should be feasible.

\section{THE FACTORS PROBLEM FOR SOFIC SHIFTS}

Problem 8.1. Suppose $S$ and $T$ are sofic shifts with $h(S) \geq h(T)$. Give necessary and sufficient conditions for the existence of a factor map from $S$ onto $T$.

As with SFTs, the factors problem for sofic shifts begins with equal and unequal entropy mixing and irreducible cases, after which there is the general case.

Suppose $T_{1}$ and $T_{2}$ are equal entropy irreducible sofic shifts, and suppose $T_{2}$ is almost finite type (AFT). (The AFT shifts are those subshifts which are the image of an irreducible SFT by a factor map which is one-to-one on a nonempty open set. The AFT shifts are a large, interesting and relatively tractable class of sofic shift $[127,130]$.

Let $p_{1}: S_{1} \rightarrow T_{1}$ and $p_{2}: S_{2} \rightarrow T_{2}$ be the canonical right closing covers of $S$ and $T$ by irreducible SFTs. Then from [37] one sees that there is a factor map from $T_{1}$ onto $T_{2}$ if and only if there is a factor map from $S_{1}$ onto $S_{2}$ whose quotient relation contains the quotient relation for $p_{1}$. We do not know when there is such a map $S_{1} \rightarrow S_{2}$. If $T_{2}$ is not AFT, we have various sufficient conditions for existence 
of a factor map $T_{1} \rightarrow T_{2}$ but I do not know conjectural necessary and sufficient conditions.

The case of Problem 8.1 where $S$ and $T$ are irreducible with $h(S)>h(T)$ is far more complicated than the corresponding problem for SFTs, and is open. The best results to date are due to Klaus Thomsen [184], who introduces a novel idea of "irreducible component" for subshifts which clarifies the factors problem.

\section{The Good Finitary Conjecture for Markov shifts}

By a mixing Markov shift in this section we mean a pair $(S, \mu)$ where $S$ is a mixing SFT and $\mu$ is an $S$-invariant Markov measure. Two such shifts $(S, \mu),\left(S^{\prime}, \mu^{\prime}\right)$ are measurably isomorphic if there is a shift commuting measurable bijection $\phi$ such that $\phi \mu=\mu^{\prime}$ and $\phi S=S^{\prime} \phi \mu$-a.e. From a classification of mixing Markov shifts up to measurable isomorphism, one easily obtains a classification of irreducible Markov shifts up to measurable isomorphism.

Following Ornstein's isomorphism theorem for Bernoulli shifts, Ornstein and Friedman [78] showed that the mixing Markov shifts were classified up to measurable isomorphism by entropy. There naturally followed explorations of various stronger equivalence relations, in which additional conditions were imposed on the measurable isomorphism $\phi$. In particular, a finitary isomorphism is a measurable isomorphism $\phi$ such that $\phi$ and $\phi^{-1}$ are continuous in the relative topology after restriction to full-measure sets. (For subshifts this means that $\phi$ and its inverse are defined on measure 1 sets by variable length block codes.) Keane and Smorodinsky [100] showed that for finite state Markov shifts, entropy is a complete invariant of finitary isomorphism.

A general finitary map is not as nasty as a general measurable map, but it may still retain a certain infinite and oracular quality. This led Parry to introduce the idea of finite expected coding time [156]. Given the finitary isomorphism $\phi$ between subshifts, one can define a.e. a coding time function $n(x)$ on its domain: $n(x)$ is the smallest nonnegative integer $n$ such that for almost all $y$, if $x[-n, n]=y[-n, n]$ then $(\phi x)_{0}=(\phi y)_{0}$. The integral of $n$ is the expected coding time. Two Markov shifts are isomorphic with finite expected coding time if they are finitarily isomorphic by a map $\phi$ such that $\phi$ and its inverse each have finited expected coding time. Schmidt introduced a related but distinct condition $[175,176]$ : a measurable isomorphism of subshifts is hyperbolic structure preserving if it respects the stable and unstable relations on sets of full measure. (That is, for all $x, y$ in some set of measure 1 in the domain of $\phi$, there exists $N \in \mathbb{Z}$ such that $x_{i}=y_{i}$ for all $i>N$ if and only if there exists $N^{\prime} \in \mathbb{Z}$ such that $(\phi x)_{i}=(\phi y)_{i}$ for all $i>N^{\prime}$; and the same holds with " $<$ " in place of ">".)

For each of these relations, the same necessary conditions for isomorphism are known. There are currently two approaches to this problem; the more developed approach involves positive elements of ideals in polynomial rings [145, 72, 73], and the other involves coding arguments on countable state Markov chains [26, 84]. Each (when it works) produces an isomorphism $\phi$ for which there is a word $W$ (of length $|W|=n$, say) such that for all $x$ in a measure 1 subset of the domain of $\phi$, if $x[i, i+|U|+2 n-1]=W U W$, then $(\phi x)[i+n-1, i+n-1+|U|]$ is determined by the word $W U W$; and likewise there is such a word for $\phi^{-1}$. Such codes are called magic word isomorphisms, and these are the codes which we define in this survey to be "good finitary isomorphisms". The magic word isomorphisms in particular 
have exponentially fast coding time and are hyperbolic structure preserving. The problem of classifying mixing Markov shifts up to good finitary isomorphism would have a very satisfactory solution if the following "Good Finitary Conjecture" were proved.

Conjecture 9.1. There is a magic word isomorphism between two mixing Markov shifts if and only if the following conditions are satisfied.

(1) They have the same beta function.

(2) They have the same ratio group $\Delta$.

(3) Their groups $\Gamma / \Delta$ (weights group modulo ratio group) have the same canonical generator.

We recommend [136] for an excellent introduction to this topic and its literature, and then further deveopments in later papers of Tuncel and collaborators (e.g. [137]). Here we'll define the invariants cited in the conjecture ("the Greeks"), which were developed in various works by Krieger, Parry, Schmidt and Tuncel.

Suppose $P$ is a stochastic matrix presenting a Markov shift. Let $P^{(t)}$ be the matrix obtained by replacing each nonzero entry $p$ of $P$ with the function $p^{t}(t \in$ $\mathbb{R})$. Let $\mathcal{R}$ be the ring of functions which are linear combinations of functions $p^{t}$ $(0<p \in \mathbb{R})$. Set $q(z)=\operatorname{det}\left(z I-P^{(t)}\right)$. Let $b(z)$ be the irreducible element of $\mathcal{R}[z]$ which divides $q(z)$ (often $q=b$ ) such that $b(1)=0$. Then the stochastic zeta function of this Markov shift is $1 / q(z)$ and Tuncel's beta function is $1 / b(z)$. The stochastic zeta function in a familiar formalism encodes the number of periodic points of period $n$ and weight $w$, for all $n$ and $w$; the beta function is analogous here to entropy. (Again, see [136].)

If $i_{0}, i_{1}, \ldots, i_{n}$ index rows/columns of $P$ such that $P\left(i_{0}, i_{1}\right) P\left(i_{1}, i_{2}\right) \cdots P\left(i_{n-1}, i_{n}\right)=$ $b>0$, then the positive real number $b$ is the weight of the path $i_{0} i_{1} \cdots i_{n}$ of length $n$. The path is a loop if $i_{0}=i_{n}$. The group $\Gamma$ is the subgroup of the multiplicative reals generated by the weights of loops. The group $\Delta$ is the set of all positive real numbers $x$ such that $x$ is the ratio of the weights of two paths of equal length and equal initial and terminal vertex. It turns out that the quotient group $\Gamma / \Delta$ is cyclic with a canonical generator.

Historically, good finitary isomorphisms between finite state Markov shifts were constructed from almost topological conjugacies: one-to-one almost everywhere extensions to a common finite state Markov shift. However, an example of Marcus and Tuncel [136] shows that it is possible for there to be a good finitary isomorphism between two mixing Markov shifts when the two shifts are not almost topologically conjugate.

\section{What CAN Be a Beta FunCtion?}

Up to topological conjugacy, a mixing SFT is defined by a primitive matrix $A$ over $\mathbb{Z}_{+}$. Here the entropy of the SFT is the $\log$ of the spectral radius $\lambda_{A}$ of $A$ and the zeta function is $\zeta(z)=(\operatorname{det}(I-A))^{-1}$. We know what these can be. Lind characterized these spectral radii: they are the Perron numbers, positive algebraic integers strictly greater in modulus than any algebraic conjugate. Kim, Ormes and Roush proved the Spectral Conjecture for $\mathbb{Z}$, characterizing the possible zeta functions. 
For a mixing SFT with a Markov measure (i.e. a mixing Markov shift), the situation is quite different. The analogue of the zeta function is the stochastic zeta function; the analogue of the entropy is the beta function (discussed in Section 9).

Problem 10.1. Characterize the functions which can be the stochastic zeta functions of mixing Markov shifts.

Problem 10.2. Characterize the functions which can be beta functions of mixing Markov shifts.

I am not aware of any conjecture addressing these problems.

These problems can be made more concrete. In section 9 , from a primitive stochastic matrix $P$ a primitive matrix $A$ over $\mathcal{R}$ was constructed to present a mixing Markov shift. One can identify several variables $x_{i}$ with chosen free abelian generators for the subgroup of the multiplicative reals generated by the positive entries of $P$, and then pass from $A$ to a matrix whose entries are Laurent polynomials in several variables with nonngative integral coefficients. The two problems above can then be restated in terms of such polynomial matrices. See [136], for an introduction to these ideas; [157, 188], which address the issue of a suitable choice of basis; and [187], which takes a several-complex-variables approach.

For a taste of the difficulties involved in such polynomial realization problems, and their applications to coding problems for Markov shifts, consult [4, 88], and [138].

\section{Structure of EXPANSIVE SUbDynamics}

We first recall some background from [41]. Let $\alpha$ be a $\mathbb{Z}^{2}$ action by homeomorphisms on an infinite compact metric space $X$. A subset $E$ of $\mathbb{R}^{2}$ is an expansive set for the action if there exist $M>0$ and $\epsilon>0$ ( $M$ and $\epsilon$ are allowed to depend on $E$ ) such that for all distinct $x, y$ in $X$, there exists $\mathbf{n} \in \mathbb{Z}^{2} \operatorname{such}$ that $\operatorname{dist}(x, E)<M$ and $\operatorname{dist}\left(\alpha^{\mathbf{n}}(x), \alpha^{\mathbf{n}}(y)\right)>\epsilon$. Let $\mathrm{E}_{1}(\alpha)$ denote the set of $\mathbf{n} \in \mathbb{R}^{d}$ such that the line $\mathbb{R} \mathbf{n}$ is an expansive set for the action. The set $\mathrm{E}_{1}(\alpha)$ is the union of open cones in $\mathbb{R}^{2}$, and there are nonzero elements of $\mathbb{R}^{2}$ in its complement. (In [41] this approach is extended to higher rank actions and higher-dimensional subspaces; for simplicity, we do not consider these here.)

For an example, suppose $B, C$ are matrices in $\operatorname{GL}(n, \mathbb{Z})$, and for $1 \leq i \leq n$ they have respectively eigenvalues $\beta_{i}, \gamma_{i}$ on a common eigenline $L_{i}$, with these lines spanning $\mathbb{R}^{n}$. Let $\alpha$ be the $\mathbb{Z}^{2}$ action on $\mathbb{T}^{n}$ defined by letting $(m, n)$ act by $B^{m} C^{n}$. Then $\mathrm{E}_{1}(\alpha)$ is the complement of the lines in $\mathbb{R}^{2}$ through the origin with slopes $-\log \left|\beta_{i}\right| / \log \left|\gamma_{i}\right|, 1 \leq i \leq n$.

An expansive component of directions is a connected component of the open set $\mathrm{E}_{1}(\alpha)$; it is an open cone contained in (possibly equal to) some half-space. We abuse language and say $\alpha^{\mathbf{n}}$ is in an expansive component if $\mathbf{n}$ is. For the $\mathbf{n}$ in a given expansive component, quantitative dynamical properties of $\alpha^{\mathbf{n}}$ tend to vary regularly as a function of $\mathbf{n}$, and qualitative properties tend to hold for all or none of these $\alpha^{\mathbf{n}}$. Especially, the stable and unstable relations for $\alpha^{\mathbf{n}}$ are the same for all $\mathbf{n}$ in a given component. (See [41, Sec. 5] for more of the viewpoint of expansiveness as a regularity condition.)

So, when a $\mathbb{Z}^{2}$ action has some element acting expansively, we think of $\mathbb{R}^{2}$ splitting into pieces: there are open cones $C$ (the expansive components) in which the dynamics of $\alpha^{\mathbf{n}}$ varies regularly with $n$; the dynamics in different components can 
differ more significantly; and the dynamics change qualitatively at boundaries of the components.

In the case that $\alpha^{\mathbf{n}}$ is a mixing SFT, we know a great deal about the dynamics of the elements $\alpha^{\mathbf{m}}$ such that $\mathbf{m}$ is in the same expansive component $\mathcal{C}$ as $\mathbf{n}$ [41]. These $\alpha^{\mathbf{m}}$ are mixing SFTs with a common measure of maximal entropy; the entropy is a linear function of $\mathbf{m}$; they are all SFTs with the same dimension group; the shift equivalence classes are nicely parametrized; and there is still sharper matrix information available from the LR textile systems of Nasu (see Appendix C).

However, we have very limited understanding of the possible structure of components, compatibility of dynamics in different components and variation of dynamics as a parameter moves from one component to another. On these matters we state various problems in this and later sections.

Problem 11.1. What sets can equal $\mathrm{E}_{1}(\alpha)$ for a $\mathbb{Z}^{2} S F T \alpha$ ? Especially, what are the possible $\mathrm{E}_{1}(\alpha)$ when there exists $\mathbf{n}$ such that $\alpha^{\mathbf{n}}$ is SFT?

We can be more specific.

Questions 11.2. Suppose $\alpha$ is a $\mathbb{Z}^{2}$ action for which there exists $\mathbf{n}$ such that $\alpha^{\mathbf{n}}$ is SFT.

(1) Is it possible for $\alpha$ to have infinitely many expansive components?

(2) Is it possible for $\alpha$ to have an expansive component with a boundary lying on a line of irrational slope?

With one exception, it is known [41] that any nonempty closed union of lines in $\mathbb{R}^{2}$ occurs as the complement of $\mathrm{E}_{1}(\alpha)$ for some $\mathbb{Z}^{2}$ subshift $\alpha$. The unknown case is Question 11.3 below.

Question 11.3. [41] Suppose $L$ is a line of irrational slope in $\mathbb{R}^{2}$. Does there exist $a \mathbb{Z}^{2}$ action $\alpha$ on a compact metric space such that $L$ is the complement of $\mathrm{E}_{1}(\alpha)$ ?

The next question is "morally" related to Question 11.3.

Question 11.4. [41] Suppose L is a line of rational slope in $\mathbb{R}^{2}$. Does there exist $a \mathbb{Z}^{2}$ action $\alpha$ on a compact metric space such that the following hold?:

- $L$ is the complement of $\mathrm{E}_{1}(\alpha)$, and

- for all nonzero $\mathbf{n}$ in $L \cap \mathbb{Z}^{2}, \alpha^{\mathbf{n}}$ is not the identity map on $X$.

The paper [132] offered a negative answer to Question 11.4, but M. Nasu has pointed out (personal communication) that [132, Lemma 3.2] is not correct; in particular, the line of slope $2 / 5$ in the example of [132] is an additional nonexpansive line. Therefore Question 11.4 is still open.

\section{Can NONSFT EXPansive Maps COMmUte With iRREducible SFTs?}

Question 12.1. [150] Suppose $S$ is an expansive automorphism of an irreducible SFT. Must $S$ be SFT?

For this type of question, we know much more if one or both of the conditions becomes onesided. A positively expansive map commuting with a onesided SFT (or a twosided SFT) must be a onesided SFT [125, 151]. An expansive automorphism $S$ of a onesided full shift on $n$ symbols must be SFT [152]; and this SFT must be shift equivalent to a full shift on $k$ symbols [44], where $k$ ([153], generalizing [44]) is possible here if and only if the following hold: 
- a prime $p$ divides $n$ iff it divides $k$, and

- if a prime $p$ divides $n$, then $p^{2}$ divides $k$.

There is one more conjectural ingredient for the onesided picture:

Conjecture 12.2. [44] An expansive automorphism of a onesided full shift is topologically conjugate (not only shift equivalent) to a twosided full shift.

Now we turn back to the open Question 12.1, where we know the following.

(1) A simple 1996 example of Doris Fiebig shows an expansive automorphism of a reducible SFT $S$ need not be SFT [24]. (In the example, the SFT consists of two fixed points and two connecting orbits.)

(2) A strictly sofic AFT (almost finite type) shift $S$ cannot commute with a mixing SFT $T$ [24]. (The AFT sofic shifts have several characterizations and seem to be the one big, natural class of relatively well behaved sofic shifts [127, Sec. 13.1]).

(3) [154] If $\phi$ is an expansive automorphism of an irreducible SFT $S$, and $\phi$ or $\phi^{-1}$ has memory zero or anticipation zero, then $\phi$ is SFT.

The result (3) of Nasu is a major advance: it is the first general result which addresses Question 12.1 for automorphisms of an SFT which need not be in the same expansive component as the SFT itself. The paper [154] extends and also unifies the "textile systems" machinery which produces all the current affirmative results related to Question 12.1, including the onesided results.

\section{The Commuting Powers Conjecture for SFTs}

We say that two continuous maps $f, g$ can commute if there are maps $F$ and $G$ topologically conjugate respectively to $f$ and $g$ such that $F G=G F$.

Conjecture 13.1. Suppose $S$ and $T$ are mixing SFTs. Then for all large $i$ and $j$, $S^{i}$ and $T^{j}$ can commute.

I stated this conjecture in a talk at the August 2005 Northwest Dynamics Conference in Victoria, after posing it as a question in a question session at the Summer 2004 Algebraic and Topological Dynamics Activity at the Max Planck Institute. (The general problem "when can two mixing shifts of finite type commute" was [41, Problem 9.9]. The very general problem of understanding the dynamics of $S^{i} T^{j}$, when $T$ is an automorphism of an SFT $S$, was posed by Nasu in [150, p.8].)

The conjecture would not be true if the assumption of passing to powers were dropped, on account of obstructions involving low order periodic points; but there is no such periodic point obstruction to the conjecture itself. This is shown in Appendix A. When $S$ and $T$ are defined by matrices which in a suitable sense commute, then Conjecture 13.1 is true. This is explained in Appendix B and Appendix D.

It would be natural to guess that commuting SFTs must in some sense arise only from commuting matrices. For onesided SFTs this is true:

- All commuting onesided SFTs can be presented by commuting matrices over $\mathbb{Z}_{+}[150]$

- There is a refined dimension group approach putting drastic constraints on which onesided SFTs can commute ([30], following [15]).

- Commuting onesided SFTs have the same measure of maximal entropy [30]. 
All of the above are not true in general for twosided SFTs. We know this on account of a dramatic example of Nasu.

Example 13.2. [150] There are square matrices $A, B$ over $\mathbb{Z}_{+}$and an automorphism $S$ of $\sigma_{A}$ such that $S$ is topologically conjugate to the mixing SFT $\sigma_{B}$ and the following hold.

- $A=\left(\begin{array}{ll}2 & 1 \\ 1 & 1\end{array}\right)$

- $B$ has characteristic polynomial $\chi_{B}(x)=(x+1)^{2}\left(x^{3}-2 x^{2}+x+1\right)$.

These two properties imply that the ranges of the maximal entropy measures of $\sigma_{A}$ and $\sigma_{B}$ on clopen sets are different, and therefore

- $S$ and $\sigma_{A}$ do not have the same measure of maximal entropy.

The SFTs $\sigma_{A}$ and $\sigma_{B}$ are not algebraically related in any obvious way: their entropies are logs of numbers generating unrelated number fields (quadratic vs. cubic), and the ranks of their dimension groups are different (2 vs. 5 ). The SFTs $\sigma_{A}, \sigma_{B}$ cannot be related to commuting matrices in the manner of Appendix D.

Nasu has developed an extensive apparatus ("textile systems" [150]) for studying the dynamics generated by a SFT (onesided or twosided) and a continuous map commuting with it. Within this structure, he provides an algorithm which, given an automorphism $U$ of an irreducible SFT, will find a matrix $B$ such that $\sigma_{B}$ is topologically conjugate to $U$, if $U$ is SFT. Example 13.2 above came from applying the algorithm to a natural example.

It is not uncommon in symbolic dynamics to encounter low order periodic point obstructions which when satisfied allow a general result (this happens for example in the context of factor maps [5], embeddings [122], and automorphisms [115, 116]). This pattern and Nasu's example are behind Conjecture 13.1. It might be that mixing SFTs can commute whenever they can commute on periodic points.

\section{JOINTLY INVARIANT MEASURES}

We recall a famous problem of Furstenberg [80]. We say two positive integers $p$ and $q$ are nonlacunary if they generate a nonlacunary semigroup under multiplication (i.e., there do not exist positive integers $k, m, n$ such that $p=k^{m}$ and $q=k^{n}$ ). Given $n \in \mathbb{N}$, in Problem 14.1 below we let $T_{n}$ denote the map $S^{1} \rightarrow S^{1}$ defined by $z \mapsto z^{n}$.

Question 14.1 (Furstenberg [80]). Suppose $p$ and $q$ are nonlacunary positive integers. Can there exist a nonatomic Borel probability $\mu$ on $S^{1}$, other than Haar measure, which is jointly invariant for $T_{p}$ and $T_{q}$ ?

Question 14.1 is not overtly a problem of symbolic dynamics but it is easily reformulated as a problem of finding the nonatomic Borel probabilities jointly invariant for the full shift on six symbols and a specific automorphism [172]; and it was in this setting that Rudolph and Johnson $[172,94]$ showed any counterexample $\mu$ to Conjecture 14.1 must have zero entropy with respect to both $T_{p}$ and $T_{q}$. Since the work of Rudolph and Johnson, the problems, results and methods around Question 14.1 have been generalized well beyond the symbolic dynamics setting (see [70, 129] and their references); but the essential problem left open by Rudolph and Johnson, the possible existence of nonatomic zero entropy measures jointly invariant for $T_{p}$ and $T_{q}$, is open still. 
Note that in the Example 13.2 of Nasu, we immediately see two jointly invariant measures of nonzero entropy (namely, the maximal entropy measures of $\sigma_{A}$ and $S$ ). We suggest that it may be fruitful to investigate the possibilities for jointly invariant measures of commuting SFTs.

For some special classes of cellular automata, there are measure rigidity results asserting that the only jointly invariant positive entropy measure is the uniform Bernoulli measure (see [93, 161, 173] and their references), and asymptotic randomization results asserting that for large classes of measures the Cesaro limit of iterates under the c.a. map exists and is the uniform Bernoulli measure. See $[74,131,160,161,162,163]$, especially the survey [161], and their references for results and open problems in this area.

A seminal example for the study of algebraic symbolic systems has been the Ledrappier 3-dot example. This is the closed shift invariant subgroup $X$ of $\{0,1\}^{\mathbb{Z}^{2}}$ consisting of points $x$ such that for all $\mathbf{n} \in \mathbb{Z}^{2}$,

$$
x(\mathbf{n})+x(\mathbf{n}+(0,1))+x(\mathbf{n}+(1,0))=0
$$

(where of course $\{0,1]\}$ represents $\mathbb{Z} / 2$ ). Basic questions on invariant measures remain open for this system, including the following.

Question 14.2. Is Haar measure the only invariant ergodic Borel probability on $X$ with full support?

Without the "full support" condition the answer to Question 14.2 is emphatically "no" [68]. See [69, 179, 180] and their references for more on this problem. More generally:

Problem 14.3. Determine the shift invariant Borel probabilities and subsystems of $X$.

\section{5. $\mathbb{Z}^{d}$ SFTS}

Irreducible SFTs are finite towers over mixing SFTs, and the nonwandering part of an SFT is a disjoint union of irreducible SFTs. From this structure, many problems about SFTs can be reduced to problems about nontrivial mixing SFTs, all of which have very similar qualitative properties. Invariants can be computed. The finer functorial problems can generally be formulated as attackable matrix problems.

The situation is very different for $\mathbb{Z}^{d}$ SFTs for $d>1$. Various problems become undecidable, exact computations are rare and the range of qualitative possibilities expands dramatically (see e.g. $[52,165,178,128]$ and their references). In contrast to the situation for $\mathbb{Z}$ SFTs, where we often have mature conjectures for refined problems, in the case of $\mathbb{Z}^{d}$ SFTs with $d>1$ we are still discovering the landscape.

Recent results of Hochman and Meyerovitch [90, 91] have recast the dynamical possibilities for $\mathbb{Z}^{d}$ SFTs $(d>1)$ in a more computation-theoretic and constructive framework. This is a strong statement, as the computation-theoretic viewpoint is by no means new here $[97,178]$; there have been striking undecidability results for $\mathbb{Z}^{d}$ SFTs and the closely related cellular automata; and the papers [90, 91] themselves rely heavily on work of Shahar Mozes and Raphael Robinson [146, 169]. However, it seems fair to say that the new constructive methods of $[90,91]$ have produced structural results at a level unprecedented in this area. For example, for any $d>1$, the possible entropies of $\mathbb{Z}^{d}$ SFTs are precisely the right recursively enumerable 
nonnegative real numbers [90]. Also, for $d>2$ there are very strong results toward the classification of $d$-dimensional cellular automata and the directional dynamics of $\mathbb{Z}^{d}$ SFTs [91]. The new constructive methods also allow hope for progress on various other problems for which there were simply no available methods.

All the constructions in $[90,91]$ are constructions of SFTs with isometric factors, and factors of such SFTs, and this underlines the potential significance of mixing conditions. In particular, what can be said about the structure of $\mathbb{Z}^{d}$ SFTs with completely positive entropy? (In the abelian algebraic case, these are weakly algebraically equivalent to Bernoulli group shifts [46].) Also, at this time the striking results in [91] on $\mathbb{Z}^{d}$ SFT subdynamics for $d \geq 3$ are not known for $d=2$, and the entropies of injective and surjective $d$-dimensional cellular automata are not characterized, especially in the case $d=1$.

Among the many open problems in this area, we mention the following question of Benjy Weiss, and a closely related question of Klaus Schmidt.

Question 15.1 (Benjy Weiss, early 90's). For $d>1$, must a $\mathbb{Z}^{d}$ sofic shift be a factor of some $\mathbb{Z}^{d}$ SFT of equal entropy?

Question 15.2 (Klaus Schmidt, late 90's). For $d>1$, if $\phi$ is a continuous factor map from a $\mathbb{Z}^{d}$ SFT $X$ onto a $\mathbb{Z}^{d}$ sofic shift $Y$, must there exist a sofic subshift $W$ of $X$ such that $h(W)=h(Y)$ and $\phi(W)=Y$ ?

For $d=1$, the answer to both questions is yes (see [127] for Question 15.1 and [135] for Question 15.2). The answer to Question 15.2 would be "no" even for $d=1$ if $W$ were required to be SFT [135].

For $d>1$, it was only recently proved that every $\mathbb{Z}^{d}$ sofic shift is a factor of an SFT of arbitrarily close entropy, and likewise that the answer to Question 15.2 would be "yes" if $W$ were required only to be arbitrarily close in entropy to $Y$ [63].

\section{Stable limit sets of Cellular automata}

We consider in this section a one dimensional cellular automaton map $f$, that is, a block code $f$ from some some full shift $X=X_{N}=\{0,1, \ldots, N-1\}$ into itself. If $f^{k}(X)=f^{k+1} X$ for some $k>0$, then $f$ has a stable limit set, and it is the sofic shift $f^{k}(X)$.

Problem 16.1. (Maass) Characterize the stable limit sets of one-dimensional cellular automata.

We will recapitulate the state of this problem, following [130]. An SFT $Y$ is a stable limit set for some c.a. map $f: X_{N} \rightarrow X_{N}$ if and only if it is mixing, contained in $X_{N}$, and contains a fixed point.

More generally, for a sofic $Y$ in $X_{N}$ to be a stable limit set of a c.a. map, it is necessary that $Y$ be mixing and contain a fixed point which is "receptive" (an easily checked technical condition). The converse holds for AFT sofic shifts but is open in general. The problem reduces to the following coding question.

Problem 16.2. Let $T$ be a mixing sofic shift with a receptive fixed point. When does there exist a block code $f$ from $T$ to $T$, and an $S F T T^{\prime}$ containing $T$, such that $f$ maps $T^{\prime}$ into $T$ ?

The following problem seems to be the heart of the matter.

Problem 16.3. Let $f$ be a surjective block code from a mixing sofic shift $T$ to itself. When does there exist an SFT $T^{\prime}$ containing $T$ such that $f$ maps $T^{\prime}$ into $T$ ? 


\section{Algebraic $\mathbb{Z}^{d}$ SFTs}

One motivation for the very successful $[177,178]$ investigation of $\mathbb{Z}^{d}$ actions of algebraic origin was to obtain some large but understandable class of $\mathbb{Z}^{d}$ actions.

By a $\mathbb{Z}^{d}$ group shift, we will mean an expansive action $\alpha$ of $\mathbb{Z}^{d}$ by continuous automorphisms of a zero dimensional compact metrizable group $X$. By a Bernoulli $\mathbb{Z}^{d}$ group shift, we will mean the $\mathbb{Z}^{d}$ shift on the product group $G^{\mathbb{Z}^{d}}$, where $G$ is a finite group with discrete topology. A group subshift of a Bernoulli shift is the restriction of the shift to a shift-invariant compact subgroup. Any group shift is algebraically conjugate (i.e., topologically conjugate by a homeomorphism which is also a group isomorphism) to a group subshift of a Bernoulli group shift.

Two group shifts are weakly algebraically equivalent [71] if each is a quotient (factor) of the other by a continuous group homomorphism. A group shift has completely positive entropy if as a measurable system with respect to Haar measure it has no nontrivial factor of zero entropy. Any abelian $\mathbb{Z}^{d}$ group shift is weakly algebraically equivalent to a unique (up to isomorphism of the alphabet group) $\mathbb{Z}^{d}$ Bernoulli group shift; any abelian $\mathbb{Z}^{d}$ group shift factors algebraically onto a Bernoulli group shift; and any (possibly nonabelian) $\mathbb{Z}^{d}$ group shift factors topologically onto a Bernoulli group shift [46].

There is a rich algebraic theory for the dynamics of actions of $\mathbb{Z}^{d}$ by continuous automorphisms of compact abelian groups [177]. The nonabelian case is much less well understood. Here in the symbolic setting are two questions.

Question 17.1. Does every nonabelian group shift factor algebraically onto a Bernoulli group shift?

Question 17.2. Is every nonabelian group shift weakly algebraically equivalent to a Bernoulli group shift?

For $d=1$, every $\mathbb{Z}^{d}$ group shift of completely positive entropy is topologically (not in general algebraically [177]) conjugate to a Bernoulli group shift [118]. Even for abelian group shifts, this is not true for $d>1[46,120]$.

Problem 17.3. Classify group shifts (especially, abelian group shifts with completely positive entropy) up to topological conjugacy.

\section{Finitary CODES AND MARKOV RANDOM FIELDS}

Suppose for $i=1,2$ that $S_{i}$ is a $\mathbb{Z}^{d}$ subshift with shift-invariant Borel probability $\mu_{i}$. A factor map $\phi$ from $\left(S_{1}, \mu_{1}\right)$ to $\left(S_{2}, \mu_{2}\right)$ is a measure-preserving map commuting with the $\mathbb{Z}^{d}$ shift action. The factor map is finitary if there is a set $N_{1}$ in $S_{1}$ such that $\phi$ is continuous (in the relative topology) on the complement of $N_{1}$. (This means that for every $x$ in the complement of a null set, there is an $M$ depending on $x$ such that the restriction of $x$ to the finite configuration $\left\{\mathbf{v} \in \mathbb{Z}^{d}:\|\mathbf{v}\| \leq M\right\}$ determines $\left.(f(x))_{0}.\right)$ If $\phi$ is an invertible finitary factor map, and its inverse is finitary, then $\phi$ is a finitary isomorphism of the measure preserving systems $\left(S_{i}, \mu_{i}\right)$, and these systems are finitarily isomorphic.

By a Bernoulli shift, we will mean a $\mathbb{Z}^{d}$ subshift with a measure whose coordinate projections are i.i.d. onto a finite state space, that is, a measure which is product measure on that finite state space. (Caveat: often, as in [13], a $\mathbb{Z}^{d}$ action with respect to some measure is called Bernoulli if it is measurably isomorphic to what we are calling a Bernoulli shift; also, while considering subshifts, we are ignoring 
infinite entropy Bernoulli systems.) For $d=1$, Keane and Smorodinsky [100] showed that every mixing Markov shift $(S, \mu)$ is finitarily isomorphic to a Bernoulli shift. For $d>1$, the analogous situation is not nearly so homogeneous.

Given a $\mathbb{Z}^{d}$ subshift $X$ with alphabet $\mathcal{A}$, and $C$ a subset of $\mathbb{Z}^{d}$, the restriction map $x \mapsto x \mid C$ maps $X$ onto a subset $X_{C}$ of $\mathcal{A}^{C}$. Given a measure $\mu$ on $X$, and $B, C$ in $\mathbb{Z}^{d}$, let $\mu(B \mid C)$ denote the conditional distribution of $\mu$ on $X_{B}$ given the distribution on $X_{C}$. If $B$ is a finite subset of $\mathbb{Z}^{d}$, let the boundary of $B$ be the set

$$
\partial B:=\left\{\mathbf{v} \in \mathbb{Z}^{d} \backslash B: \exists \mathbf{w} \in B,\|\mathbf{w}-\mathbf{v}\|_{1}=1\right\} .
$$

A Markov random field is a measure $\mu$ on a $\mathbb{Z}^{d}$ shift $S$ with the following property: for every finite subset $B$ of $\mathbb{Z}^{d}$ (with complement $B^{\prime}$ ) and $\mu$-almost all $x$ in $S$, the conditional distributions $\mu\left(B \mid B^{\prime}\right)$ and $\mu(B \mid \partial B)$ are equal. (We are only considering Markov random fields with finite state space, that is, the stochastic process of coordinate projections has finite range.) The random field is stationary if the measure is invariant under the $\mathbb{Z}^{d}$ shift. Unless stated otherwise, by Markov random field we will mean stationary Markov random field.

The $\mathbb{Z}^{d}$ generalization of a Markov measure is a Markov random field. It was shown by del Junco [95] that for any Markov random field $\mu$ on a subshift $S$, there are finitary factor maps from $(S, \mu)$ onto all Bernoulli shifts of strictly lower entropy. On the other hand, Hoffman showed that a subshift with a stationary Markov random field can be $K$ (equivalently, have no nontrivial measurable factor of zero entropy) and still not be measurably isomorphic to a Bernoulli shift [92].

Moreover, van den Berg and Steif [13] gave examples of shifts with Markov random fields which are measurably isomorphic to Bernoulli shifts (these fields can even be measures of maximal entropy), but which are not the image of a Bernoulli shift under any finitary factor map, as follows. Suppose there are distinct ergodic Markov random fields $\mu, \nu$ on a $\mathbb{Z}^{d}$ full shift $S$, assigning positive measure to every open set, such that $\mu(B \mid \partial B)=\nu(B \mid \partial B)$ for every finite subset $B$ of $\mathbb{Z}^{d}$. In this case (and for other interesting examples with the full-support condition relaxed), van den Berg and Steif showed there cannot exist a finitary factor map from a Bernoulli shift onto $(S, \mu)$.

On the other hand, Steif and van den Berg established a large class of Markov random fields which are finitary factors of Bernoulli shifts; and they showed that both scenarios occur for the Ising model, and that the two scenarios correspond to whether one is above or below the critical paramter. Thus the idea of a finitary isomorphism, which arose for $\mathbb{Z}$ actions as an abstractly natural equivalence, has for $\mathbb{Z}^{d}$ shifts an unexpected and intriguing relation to phase transitions. We mention two of the various problems in this direction.

Problem 18.1. [13, Question 3] If a translation invariant Markov random field $\mu$ on a shift $S$ is the unique Markov random field (among both translation invariant and non-translation invariant fields) with its conditional probabilities, must $(S, \mu)$ be measurably isomorphic to a Bernoulli shift?

The measure $\mu$ in Problem 18.1 must be $K$ [13].

Question 18.2 (Steif). Suppose a $\mathbb{Z}^{d}$ SFT has a unique measure of maximal entropy which is Bernoulli. Must there exist an i.i.d. process mapping finitarily onto it?

There are more questions in [13]. 


\section{Decidability problems}

There are many open decidability problems, including the following.

Problems 19.1. Determine for any of the following whether there exists a decision procedure.

(1) Given square matrices $A$ and $B$ over $\mathbb{Z}_{+}$, decide whether the associated SFTs $\sigma_{A}$ and $\sigma_{B}$ are topologically conjugate. (Equivalently, decide whether $A$ and $B$ are strong shift equivalent over $\mathbb{Z}_{+}$.)

(2) Decide whether two given sofic shifts are topologically conjugate.

(3) Decide whether two given onesided sofic shifts are conjugate. (See Section 21.)

(4) If $U$ is an expansive automorphism of an SFT $S$ (e.g. $U=S$ ), compute (for the $\mathbb{Z}^{2}$ action generated by $U$ and $S$ ) the expansive component $\mathcal{C}$ containing of $S$.

(In Nasu's theory of textile systems [150], $\mathcal{C}$ is the interior of the ELR cone for $U$, and Nasu provides an algorithm which enumerates a sequence of cones whose union will be this interior. However, we do not know a procedure which produces convergent upper bounds to this $\mathcal{C}$.)

(5) [97, Open Problem 7] Given a surjective block code $\phi$ on a full shift, decide if $\phi$ is positively expansive.

(6) Given an automorphism $\phi$ of a mixing SFT, decide if $\phi$ is expansive.

(7) Given an expansive automorphism $\phi$ of a mixing SFT, decide if $\phi$ is SFT.

(Here, Nasu provides an algorithm which, if $\phi$ is SFT, will eventually affirm $\phi$ is SFT and will return a matrix defining an edge SFT topologically conjugate to $\phi[150]$. However, if $\phi$ is expansive and not SFT, then this algorithm will never halt.)

Even for 1-dimensional cellular automata (endomorphisms of full shifts), there cannot exist a general procedure for estimating the entropy of a cellular automaton; as far as I know, though, it is unknown if such a procedure can exist for invertible one-dimensional cellular automata. For any (!) nontrivial property of the limit set (eventual image) of a cellular automaton, there cannot exist a general procedure which given a cellular automaton will decide if its limit set has the property [96]. For more on such results and problems for cellular automata, see [97, 98].

What decision procedures do we know? We can decide using periodic points whether a block code from an irreducible SFT is surjective or if it is injective. We can decide whether a subshift factor of an SFT is SFT. From work of Kim and Roush, partly in collaboration with Bratteli and Jorgenson, we can decide more or less everything related to shift equivalence of integral marices. For example, we can decide whether given matrices are shift equivalent over $\mathbb{Z}_{+}[102]$, and whether two stationary dimension groups are isomorphic [50]. For twosided sofic shifts, there is a theory of shift equivalence and strong shift equivalence [38, 87, 147] analogous to the SFT theory: two sofic shifts are shift equivalent iff they have all large powers conjugate, and they are strong shift equivalent iff they are conjugate. Kim and Roush have also shown how to decide whether two sofic shifts are shift equivalent [104]. 


\section{ONESIDED SFTS: THE EMBEDDING PROBLEM}

Given a shift $S$, let $\mathcal{O}_{n}(S)$ denote the set of periodic orbits of cardinality $n$ in the shift $S$.

Let $T$ be a mixing SFT. Krieger's Embedding Theorem [122] states that a given subshift $S$ is isomorphic to a proper subshift of $T$ if and only if the following conditions hold:

(1) for all $n,\left|\mathcal{O}_{n}(S)\right| \leq\left|\mathcal{O}_{n}(T)\right|$, and

(2) $h(S)<h(T)$.

Krieger's Embedding Theorem is tremendously useful for many constructions involving SFTs, and even for the construction of matrices with specified spectra [33]. We have no analogous result for onesided shifts.

Problem 20.1. Suppose $T$ is a mixing onesided SFT and suppose $S$ is a subshift such that $h(S)<h(T)$. Give good necessary and sufficient conditions for $S$ to be isomorphic to a subshift of $T$.

Next is the first step toward solving Problem 20.1.

Question 20.2. Suppose $S$ is a onesided subshift, $T$ is the full onesided shift on $N$ symbols, $h(S)<h(T)=\log N$, and no point of $S$ has more than $N$ preimages. Must $T$ be isomorphic to a subshift of $S$ ?

A solution to Problem 20.1 cannot have the simplicity of Krieger's Theorem. For example, if $\phi$ is a block code embedding $S$ into $T$ and $x$ is in $S$, then $\phi(x)$ cannot have more preimages in $T$ than $x$ has in $S$; and more generally, the whole infinite preimage tree of $x$ embeds into the preimage tree of $\phi(x)$. In particular, the embedding of periodic points must be compatible with an embedding of their preimage trees. Question 20.2 avoids these complications.

We remark, there is no real loss of generality in restricting these problems to the case that $S$ is SFT. This is because any $S$ is the intersection of a decreasing sequence of SFTs $S_{n}$, and $S$ embeds into a given SFT $T$ if and only $S_{n}$ embeds into $T$ for all large $n$.

\section{ONESIDED SOFIC SHIFTS: THE CLASSIFICATION PROBLEM}

Problem 21.1. Classify onesided sofic shifts.

We will see this problem has a very finite presentation - but, we really do not know whether there exists a decision procedure for the classification.

Let us consider the context for Problem 21.1. First, we recall Williams' classification for onesided SFTs [192, 32]. Let $A$ be an $n \times n$ matrix over $\mathbb{Z}_{+}$. If columns $i$ and $j$ are equal, then define an $(n-1) \times(n-1)$ matrix $A^{\prime}$ as follows: erase column $i$ to get a matrix $A^{\prime \prime}$; replace rows $i$ and $j$ of $A^{\prime \prime}$ with a single row which is the sum of its rows $i$ and $j$. For example, using $i=1$ and $j=2$ we compute

$$
A=\left(\begin{array}{lll}
1 & 1 & 1 \\
0 & 0 & 1 \\
1 & 1 & 3
\end{array}\right) \rightarrow A^{\prime \prime}=\left(\begin{array}{ll}
1 & 1 \\
0 & 1 \\
1 & 3
\end{array}\right) \rightarrow A^{\prime}=\left(\begin{array}{ll}
1 & 2 \\
1 & 3
\end{array}\right)
$$

Here $A^{\prime}$ is a one-step amalgamation of $A$. If $A^{\prime}$ has two equal columns, compute a smaller matrix which is a one-step amalgamation of $A^{\prime}$. Continue until arriving at a matrix which has all columns distinct. This matrix is the total amalgamation 
$A_{\text {tot }}$ of $A$. Up to conjugation by a permutation matrix, all choices of one-step amalgamations will produce the same $A_{\text {tot }}$. Williams showed that for matrices $A$ and $B$ without zero rows, the associated onesided SFTs are topologically conjugate if and only if there is a permutation matrix $P$ such that $A_{\text {tot }}=P B_{\text {tot }} P^{-1}[192,32]$. That is about as simple and effective as a classification can be.

Next, consider two irreducible onesided SFTs supporting Markov measures. When is there a measurable isomorphism of these measurable systems? Perhaps surprisingly, this turns out to be a question of symbolic dynamics. Instead of matrices over $\mathbb{Z}_{+}$one considers matrices $P, Q$ over a suitable larger ring. It is not known if there is an analogue of the total amalgamation, but it is known that $P, Q$ define measurably isomorphic Markov shifts if and only if there is a matrix $R$ of the same type, and not larger than $P$ or $Q$, which defines a Markov shift which is a resolving factor of the other two; this is very manageable and there is a decision procedure which given $P$ and $Q$ will produce $R$ or state $R$ does not exist. This theorem of Ashley, Marcus and Tuncel [8] is a satisfying application of symbolic dynamics to ergodic theory, and the ultimate classification is finite and effective.

Now for the sofic problem we focus on the main case, the irreducible onesided sofic shifts (i.e. the factors of irreducible onesided SFTs). For such a shift $T$, using the usual irreducible predecessors cover construction [127], one can construct an irreducible SFT $S$ and a left resolving one-block factor map $p: S \rightarrow T$ given by a labeled directed graph (a symbol of the shift $S$ is an edge of the graph, and the one-block code sends that symbol to its label). Such a labeled graph has a "labeled" adjacency matrix $M$ : e.g., if there are three edges from vertex $i$ to vertex $j$ respectively labeled $a, a, b$, then $M(i, j)=2 a+b$. Such a matrix $M$ is called irreducible if the underlying adjacency matrix over $\mathbb{Z}_{+}$is irreducible. The construction for $p$ produces an irreducible defining matrix $M$ with all columns distinct; and every irreducible matrix $M$ with columns distinct defines such a cover. Such a cover is the canonical left resolving irreducible cover of its sofic shift image.

The cover is canonical in the following sense. Suppose $p: S \rightarrow T$ and $p^{\prime}: S^{\prime} \rightarrow T^{\prime}$ are two such covers, and $\psi^{\prime}: T \rightarrow T^{\prime}$ is a topological conjugacy. Then there exists a unique topological conjugacy $\widetilde{\psi}: S \rightarrow S^{\prime}$ such that $\psi p=p^{\prime} \widetilde{\psi}$. Thus $T$ and $T^{\prime}$ are topologically conjugate if and only if there exists some topological conjugacy $\phi: S \rightarrow S^{\prime}$ which takes the quotient relation of $p$ to the quotient relation of $p^{\prime}$ (i.e., $p(x)=p(y)$ if and only if $\left.p^{\prime}(\phi x)=p^{\prime}(\phi y)\right)$ [79]. Because we can decide whether $S$ and $S^{\prime}$ are conjugate, it remains to understand the case where $S^{\prime}=S$.

Problem 21.2. Give a procedure which decides, given canonical left resolving irreducible covers $p, p^{\prime}$ from a onesided irreducible SFT $S$, whether there is an automorphism of $S$ which takes the quotient relation for $p$ to the quotient relation for $p^{\prime}$.

If the onesided SFT $S$ has as a total amalgamation matrix a matrix $A$ such that $A$ is a zero-one adjacency matrix of a directed graph with no nontrivial graph automorphism, then the only automorphism of $S$ is the identity [32]. In this case, one can simply present two covers from $S$ by labelings of a suitably high block presentation. If the labelings are not equal, then the onesided sofic shifts are not conjugate. In some other cases (e.g. $A=[2]$, or $A$ any zero-one matrix), the automorphism group of $S$ will be finite and there is again a straightforward procedure for deciding conjugacy of sofic shifts whose canonical covers have domain $S$. These results are some evidence that Problem 21.2 might be a correct approach to Problem 21.1. 
Fujiwara [79] gave an interesting and appropriate algebraic reformulation of this problem, but it has not solved Problem 21.1. By analogy with the two classifications discussed earlier, one naturally hopes for something like a classifying amalgamation. Already Fujiwara gave an example showing in that algebraic framework a total amalgamation does not generally exist; and Michael Schraudner (personal communication) has shown me an example of two $4 \times 4$ labeled matrices which define the canonical left resolving covers of topologically conjugate onesided sofic shifts, but which cannot both be conjugated by one-block codes to any cover defined by an $n \times n$ labeled matrix with $n \leq 4$.

In Appendix F, we construct a large class of examples which give further restrictions on possibilities of this type.

\section{The Virtual FOG Conjecture for automorphisms of a mixing SFT}

Let $A$ be a primitive matrix over $\mathbb{Z}$, so $S=\sigma_{A}$ is a mixing SFT. The automorphism group $\operatorname{Aut}(S)$ of a homeomorphism $S$ is the group of homeomorphisms which commute with $S$ [43]. When $S$ is a nontrivial mixing SFT, this group is a countable residually finite group, containing copies of all finite groups and many infinite groups, but not of any group with unsolvable word problem. By a theorem of Ryan, the center of the group is the group of powers of $S$. It would be interesting to have some understanding of this naturally occuring class of complicated groups, but they are mysterious. Apart from some application of Ryan's theorem, we know no way of distinguish the various $\operatorname{Aut}(S)$ as groups. For example,

Problem 22.1. Are $\operatorname{Aut}\left(\sigma_{2}\right)$ and $\operatorname{Aut}\left(\sigma_{3}\right)$ isomorphic groups?

We denote the kernel of the dimension representation $\rho$ as $\operatorname{Aut}_{0}(S)$, the group of inert automorphisms of $S$. The subgroup $\operatorname{Aut}_{0}(S)$ is combinatorially complex, and seems to have a qualitative personality independent of $S$. For example, the rich action of this subgroup on finite subsystems (and even on proper subshifts) of $S$ is now well understood $[115,116,39]$, and apart from some differences involving low order periodic points the possible actions are essentially the same. In contrast to the richness and seeming homogeneity of $\operatorname{Aut}_{0}(S)$, the range of $\rho$ is typically a finitely generated abelian group which reflects the algebra of $A$.

The mystery of the algebraic structure of $\operatorname{Aut}(S)$ lies mainly in $\operatorname{Aut}_{0}(S)$. For example, we do not know its commutator or its finite index subgroups. (We also do not know, for mixing SFTs $S$ and $T$, whether a group isomorphism $\operatorname{Aut}(S) \rightarrow$ $\operatorname{Aut}(T)$ must send $\operatorname{Aut}_{0}(S)$ to $\operatorname{Aut}_{0}(T)$.)

Let $\mathrm{F}_{0}(S)$ denote the subgroup of $\operatorname{Aut}_{0}(S)$ generated by its elements of finite order and let $Q_{0}(S)$ denote the quotient group $\operatorname{Aut}_{0}(S) / \mathrm{F}_{0}(S)$. For years, the "FOG (finite order generation) Conjecture" asserted that $Q_{0}(S)$ is trivial. We now have complete descriptions of the actions of $\mathrm{F}_{0}(S)[29]$ and $\operatorname{Aut}_{0}(S)[115,116]$ on finite subsystems of $S$, and we know that in some cases the quotient group $Q_{0}(S)$ is nontrivial.

Conjecture 22.2 (Virtual FOG Conjecture). If $S$ is a mixing SFT $S$, then the group $\operatorname{Aut}_{0}(S) / \mathrm{F}_{0}(S)$ is finite. 


\section{Topological orbit EQUivalence}

Let $\alpha, \beta$ denote actions of groups $G, H$ by homeomorphisms on compact metric spaces $X, X^{\prime}$. The actions are topologically orbit equivalent if there exists a homeomorphism $h: X \rightarrow X^{\prime}$ such that for every $\alpha$ orbit $\mathcal{O}$, the set $h(\mathcal{O})$ is a $\beta$ orbit. An action $\alpha$ is minimal if every $\alpha$-orbit is dense.

Problem 23.1. Classify minimal actions of $\mathbb{Z}^{d}$ on the Cantor set up to topological orbit equivalence.

This problem is solved (spectacularly) for $d=1$ [81]; to see the large and diverse set of papers which followed, examine the citations to [81] in MathSciNet.

Question 23.2. For $d>1$, is a minimal $\mathbb{Z}^{d}$ action on the Cantor set topologically orbit equivalent to a $\mathbb{Z}$ action?

Because the $\mathbb{Z}$ classification is known, an answer "yes" to Question 23.2 would solve Problem 23.1. The preprint [82] gives an answer "yes" to Question 23.2 in the case $d=2$. Question 23.2 is a special case of the following question motivated by analogy with Dye's Theorem in ergodic theory.

Question 23.3. Is a minimal action on the Cantor set by a countable amenable group topologically orbit equivalent to a $\mathbb{Z}$ action?

The minimal $\mathbb{Z}$ actions on a Cantor set $X$ are classified by associated unital ordered groups. The group is $C(X, \mathbb{Z})$ modulo $\operatorname{Inf}_{\alpha}(X, \mathbb{Z})$, its subgroup of infinitesimals (functions with zero integral for every $\alpha$-invariant Borel probability); an element $[f]$ of the group is in the positive set if $[f]=[g]$ for some function $g \geq 0$; and the distinguished order unit is [1], the class of the constant function 1. For the minimal actions on the Cantor set, the ordered groups appearing in this way are precisely the simple dimension groups for which the subgroup of infinitesimals is trivial and which are not $\mathbb{Z}$. (We refer to [81] for the ordered group definitions for this class.) For nonminimal homeomorphisms of zero dimensional compact metrizable spaces, these unital ordered groups are still topological orbit equivalence invariants for any homeomorphism of the Cantor set, although the ordered groups are typically no longer dimension groups; and they have been applied in $[35,117]$ to nonminimal systems. For example, the flow equivalence of irreducible shifts of finite type of positive entropy is equivalent to isomorphism of their ordered cohomology groups [35] (isomorphism respecting the distinguished units is a finer relation). The case in which the orbit equivalence is defined by a bounded coycle is much more rigid (see [48], and for nonzero dimension [76]).

Among the many open problems, we mention the following.

Problems 23.4. (1) Classify irreducible SFTs up to topological orbit equivalence.

(2) Characterize the unital ordered groups which occur as the ordered cohomology for homeomorphisms of subshifts/irreducible SFTs/compact zero dimensional spaces.

(3) Classify the unital ordered cohomology groups of irreducible SFTs and sofic systems.

(4) When $X$ is zero dimensional, the cohomology group $C(X) /(I-T) X$ carries an alternate natural order, the winding order (see [35]). When is the winding order the same as the standard order? 
(5) If an irreducible SFT is topologically orbit equivalent to a subshift $S$, must $S$ be SFT?

On these items, see $[35,117]$ and their references. Kim, Roush and Williams [117] among other contributions introduced ordered homology groups and a refinement of ordered cohomology which classifies irreducible SFTs up to homeomorphisms which send finite orbits to finite orbits (or equivalently, they show, orbit closures to orbit closures).

It is possible for mixing SFTs to be topologically orbit equivalent to nonexpansive maps; it is possible for sofic shifts to be topological orbit equivalent to nonsofic shifts; any homeomorphism $h$ defining a topological orbit equivalence of irreducible SFTs must send Markov measures to Markov measures, but $h$ may disrespect past and future on a dense $G_{\delta}$ set.

For irreducible SFTs $S$ and $T$ to be topologically orbit equivalent, the following are the known necessary conditions [35].

(1) The measures of maximal entropy of $S$ and $T$ have the same range on closed open sets.

(2) $S$ and $T$ have the same zeta function.

(3) $S$ and $T$ are flow equivalent.

On the other side, it is not known whether topological orbit equivalence of irreducible SFTs $S$ and $T$ implies that $S$ is topologically conjugate to $T$ or $T^{-1}$.

\section{Symbolic EXTENSION ENTROPY AND ENTROPY STRUCTURE}

Suppose $T$ is a homeomorphism of a compact metric space $X$, and let $\mathcal{M}_{T}$ denote the compact metrizable space of $T$-invariant Borel probabilities. If $S: Y \rightarrow Y$ is a subshift on a finite alphabet and $\varphi: Y \rightarrow X$ is a continuous surjection such that $\varphi S=T \varphi$, then we we say that $\varphi$ is a symbolic extension of $(X, T)$. The extension entropy function of $\varphi$ is the function $h_{\mathrm{ext}}^{\varphi}$ on $\mathcal{M}(T)$ defined by the rule

$$
h_{\mathrm{ext}}^{\varphi}: \mu \mapsto \max \left\{h_{\nu}(S): \nu \in \mathcal{M}(S), \varphi \nu=\mu\right\} .
$$

The symbolic extension entropy function $h_{\text {sex }}^{T}$ is the infimum of the extension entropy functions $h_{\mathrm{ext}}^{\varphi}$, taken over all symbolic extensions $\varphi[27]$. (If there is no symbolic extension, then we set $h_{\mathrm{sex}}^{T} \equiv \infty$. Also, we use "sex entropy" as an abbreviation for "symbolic extension entropy".) The function $h_{\mathrm{sex}}^{T}$ is a very fine reflection of how and where complexity emerges in $(X, T)$ along refining scales, and underpins the Downarowicz theory of entropy structure [64]. The entropy structure of $T$ is an equivalence class of certain sequences of real valued functions on the $\mathcal{M}(T)$; see [64] for the definition and motivation. Suffice it to say that entropy structure is a master invariant for entropy theory, which determines in particular the entropy function on $\mathcal{M}(T)\left(\mu \mapsto h_{\mu}(T)\right)$ and the symbolic extension entropy function $h_{\mathrm{sex}}^{T}$.

A related invariant is $\mathbf{h}_{\mathrm{sex}}(T)$, the topological sex entropy of $T$. This is the infimum of the topological entropies of $(Y, S)$, taken again over all symbolic extensions $\varphi:(Y, S) \rightarrow(X, T)$. If $T$ is $C^{\infty}$, then $\mathbf{h}_{\mathrm{sex}}(T)=h_{\mathrm{top}}(T)$ [31, Theorem 7.8]. If $T$ is only $C^{1}$, then $\mathbf{h}_{\text {sex }}(T)$ can be infinite (i.e., there is no symbolic extension) [65]. For $1<r<\infty$, it is not known whether there exists a $C^{r}$ system $(X, T)$ with $\mathbf{h}_{\text {sex }}(T)=\infty$. In [65], it is shown that within certain families of $C^{r}$ systems $(1<r<\infty), \mathbf{h}_{\text {sex }}(T)>h_{\text {top }}(T)$ is a generic property. Concrete $C^{r}$ examples and functorial properties of $h_{\text {sex }}$ are examined in [28]. 
Problem 24.1. [65] Is it possible for a $C^{r}$ diffeomorphism of a compact Riemannian manifold to have infinite symbolic extension entropy?

Problem 24.2. [89, Question 6.7] Given $1 \leq r \leq \infty$, which entropy structures can occur for $C^{r}$ diffeomorphism of a compact Riemannian manifold?

Problem 24.2 is perhaps a program for the decades rather than a single problem. However, already Downarowicz and Serafin [66] have explained exactly which sequences of functions can occur as an entropy structure for a homeomorphism of a compact metrizable space, and showed all of them can be realized on zero dimensional spaces. For other specific instances of Problem 24.2, see [89, Section $6]$.

\section{Cellular automata AND Periodic points}

Given $\mathcal{A}$ a set of $n$ elements and $d$ a positive integer, let $X=\mathcal{A}^{\mathbb{Z}^{d}}$. The natural shift action of $\mathbb{Z}^{d}$ defines the full $\mathbb{Z}^{d}$ shift on $n$ symbols. A $d$-dimensional cellular automaton is a map $f$ from some such $X$ to itself, defined by a block code. A point $x$ is periodic for $f$ ("temporally periodic") if there exists $k>0$ such that $f^{k}(x)=x$. A point is periodic for the shift ("spatially periodic") if its orbit under the shift is finite. A point is jointly periodic if it is periodic for both $f$ and the shift.

Question 25.1. [17, 36, 97] Must a d-dimensional surjective cellular automaton map have dense periodic points? dense jointly periodic points?

(Joe Auslander followed this question with a related one - must the minimal subsystems of a surjective cellular automaton map be dense?)

Question 25.1 has been studied for $d=1$ dimensional c.a., but so far as I know it is also open for $d>1$. In the case $d=1$, the answer is "yes" if $f$ is an algebraic, closing or equicontinuous map $[36,17]$. There is also some experimental evidence [40] in the case $d=1$, and in this case the question is a conjecture:

Conjecture 25.2. [40] For every surjective 1-dimensional cellular automaton, the jointly periodic points are dense.

One would like some quantitative understanding about the frequency of the jointly periodic points within the spatially periodic points. We will mention three problems (two questions and a conjecture) which indicate how badly we lack techniques for this. Let $S_{N}$ denote the one-dimensional full shift on $N$ symbols. Given a one-dimensional cellular automaton $f$ and $k \in \mathbb{N}$, for the moment let $P$ be the number of fixed points of $\left(S_{N}\right)^{k}$ which are also periodic for $f$, and let $\nu_{k}\left(f, S_{N}\right)=P^{1 / k}$. Set

$$
\nu\left(f, S_{N}\right)=\limsup _{k} \nu_{k}\left(f, S_{N}\right)
$$

Question 25.3. [40] Is it true for every surjective one dimensional cellular automaton $f$ on $N$ symbols that $\nu\left(f, S_{N}\right)>1$ ?

Question 25.4. [40] Is it true for every surjective one dimensional cellular automaton $f$ on $N$ symbols that $\nu\left(f, S_{N}\right) \geq \sqrt{N}$ ?

Conjecture 25.5. [40] There exists $N>1$ and a surjective one-dimensional cellular automaton $f$ such that $\nu\left(f, S_{N}\right)<N$. 
It seems perfectly clear from the experimental evidence [40] that there are many c.a. satisfying the inequality of Conjecture 25.5.

Periodic points of $d$-dimensional cellular automata $f$ become less constrained for $d>1$. We copy below an open problem of Kari in this area. There, $P$ is the set of spatially periodic points, and $f_{P}$ is the map $P \rightarrow P$ given by restriction of $f$. In the case that there is a given "quiescent state" $q$ (i.e. the point $x$ such that $x(\mathbf{n})=q$ for all $\mathbf{n} \in \mathbb{Z}^{d}$ is a fixed point of $\left.f\right), F$ is the set of points $x$ such that $x(\mathbf{n})=q$ for all but finitely many $\mathbf{n}$ in $\mathbb{Z}^{d}$, and $f_{F}: F \rightarrow F$ is again given by restriction.

Questions 25.6. [97, Open Problem 2] For a d-dimensional cellular automaton $f$, with $d \geq 2$,

(1) does injectivity of $f_{P}$ imply surjectivity of $f_{F}$ ?

(2) does surjectivity of $f_{F}$ imply surjectivity of $f_{P}$ ?

(3) does surjectivity of $f$ imply surjectivity of $f_{P}$ ?

For $d=1$ the answer to all of these questions is "yes".

\section{Cellular automata on Big Groups}

Let $G$ be a discrete countable group. One can consider a finite set $A$ and the product space $A^{G}$ and the shift action by $G$, where for $x \in A^{G}$, the point $\sigma^{g} x$ is defined by $\left(\sigma^{g} x\right)(h)=x(h g)$. The full shifts already described for $G=\mathbb{Z}^{d}$ are a special case of this construction. A group $G$ is surjunctive if for all $A$ a continuous shift commuting map $A^{G} \rightarrow A^{G}$ must be either surjective or noninjective.

Problem 26.1 (Gromov [85]). Which groups are surjunctive?

Gromov introduced sofic groups [85], a class which generalizes amenable and residually finite groups. Weiss proved that the sofic groups are surjunctive [191].

There are many papers in this area, including $[59,60,75]$ and their references.

\section{Entropy conjugacy and Countable state Markov Chains}

Let $S, T$ be Borel isomorphisms of standard Borel spaces. Define the entropy $h(S)$ of $S$ as the supremum, over the $S$-invariant Borel probabilities $\mu$, of the measure theoretic entropies $h_{\mu}(S)$. Let $\mathcal{M}^{\text {erg }}(a, S)$ denote the $S$-invariant ergodic Borel probabilities $\mu$ such that $h_{\mu}(S) \geq a$. Following Buzzi [53,26], say that $S$ and $T$ are entropy conjugate if there is a number $a$, with $0 \leq a<h(S)=h(T)$, and a Borel isomorphism $\phi$ from the domain of $S$ to the domain of $T$, such that $\phi$ induces a bijection $\mathcal{M}^{\text {erg }}(a, S) \rightarrow \mathcal{M}^{\text {erg }}(a, T)$ which for each $\mu \in \mathcal{M}^{\text {erg }}(a, S)$ restricts to a measurable conjugacy of the measurable systems $(S, \mu)$ and $(T, \phi \mu)$.

Question 27.1. Suppose $S$ and $T$ are positive recurrent, mixing countable state Markov shifts of equal entropy. Must $S$ and $T$ be entropy conjugate?

The answer to Question 27.1 is "yes" if the two shifts are strongly positive recurrent [26], and by previous work of Buzzi this has applications to certain classes of smooth or piecewise smooth systems [26]. It would be interesting to have a better idea of how general this kind of phenomenon is. 


\section{Beta ShIFTS: SAlEM NUMBERS AND INTRINSIC ERGODICITY}

For $\beta>1$, the $\beta$ transformation on $[0,1)$ is the piecewise linear mapping $T_{\beta}$ which sends $x$ the fractional part of $\beta x$. Their study goes back now some 50 years. Let $\operatorname{Per}(\beta)$ denote the periodic points of $T_{\beta}$.

Klaus Schmidt $[174,19]$ showed that when $\beta$ is a $\operatorname{Pisot}$ number, $\operatorname{Per}(\beta)=\mathbb{Q} \cap$ $[0,1)$; and if $\operatorname{Per}(\beta)=\mathbb{Q} \cap[0,1)$, then $\beta$ is either a Pisot or a Salem number.

Conjecture 28.1 (Schmidt, 1980). $\operatorname{Per}(\beta)=\mathbb{Q} \cap[0,1)$ if $\beta$ is a Salem number.

This conjecture is just naturally interesting, but the importance of Salem numbers for Lehmer's Conjecture adds extra spice; something difficult about Salem numbers is potentially significant.

The $\beta$ transformation has a natural cover by a subshift known as the $\beta$ shift; the family of $\beta$ shifts is a rich source of examples. The following problem for $\beta$ shifts is much newer. Recall a system is intrinsically ergodic if it has a unique measure of maximal entropy.

Problem 28.2. [185] [Klaus Thomsen] Must a subshift factor of a $\beta$ shift be intrinsically ergodic?

The $\beta$ shifts themselves are intrinsically ergodic and mixing.

\section{AdleR's Renewal Question}

A subshift is a renewal shift if there is a finite set of words such that domain of the subshift is the set of all doubly infinite concatenations of such words.

Question 29.1 (Adler, 1980's). Is every irreducible sofic shift topologically conjugate to a renewal shift?

Goldberger, Lind and Smorodinsky [83] shows that all entropies of sofic shifts were entropies of renewal shifts. Adler's intuition was that the question might be related to the conjugacy problem for SFTs (which is not known to be decidable).

\section{The Road Coloring Problem}

Problem 30.1 (The Road Coloring Problem). Let $G$ be an irreducible directed graph such that every vertex has the same number $n$ of outgoing edges. Must there exist a coloring of the edges of $G$ with $n$ colors satisfying the following?:

(1) at each vertex, the $n$ outgoing edges have distinct colors, and

(2) there exists $k \in \mathbb{N}$ and a finite string of colors $c=c_{1} \ldots c_{k}$ such that all paths of length $k$ in $G$ with the coloring $c$ end at the same vertex.

The name for the problem (which we will refer to simply as the Road Problem) comes from thinking of the vertices of $G$ as intersections of colored roads, and the string $c$ as a map. If the road problem has a solution, then you can follow the map home regardless of where you start. The Road Problem was proposed as a target lemma for proving a theorem of symbolic dynamics; Adler, Goodwyn and Weiss found another method for that theorem [1].

The significance of the Road Problem for dynamics itself is perhaps no longer so clear (although, a very natural problem for the classification of onesided Markov chains is known to be strictly more difficult than the Road Problem [8]). But 
as a simply stated, longstanding problem with roots in dynamics, its inclusion is irresistible.

See $[51,54]$ and their references for progress on and generalizations of the Road Problem. ADDENDUM: The Road Coloring Problem has been solved by A. Trahtman [186].

\section{Parry's Finiteness Question for Skew PRODUCTS}

This is a problem posed by Bill Parry some time in the 90's, to which he devoted a good deal of effort in years following 2001.

Consider a finite abelian group $G$; an irreducible SFT $\sigma_{A}$ with domain $X_{A}$; and a continuous function $f$ from $X_{A}$ into $G$. The weight of $G$ on a finite orbit of $\sigma_{A}$ is the sum of $f(x)$ over $x$ in the orbit. After passage to a higher block presentation system of the SFT, the function $f$ can be presented by a $G$-labeled directed graph; this labeled graph has an adjacency matrix $B$ with entries in $\mathbb{Z}_{+} G$, the positive cone of the integral group ring of $G$; the sequence $\left(\operatorname{trace}\left(B^{n}\right)\right)_{n \in \mathbb{N}}$ encodes for all $n$ in $\mathbb{N}$ and $g \in G$ the number of $\sigma_{A}$ orbits of cardinality $n$ with weight $g$ and this sequence in standard fashion is itself encoded as a zeta function

$$
\zeta_{f}(z)=\sum_{n=1}^{\infty} \frac{\operatorname{trace}\left(B^{n}\right)}{n} z^{n}=\zeta_{f}(z)=1 / \operatorname{det}(I-z B) .
$$

A function $f$ as above defines a skew product; its domain is $X_{A, f}=X_{A} \times G$, and the homeomorphism $X_{A, f} \rightarrow X_{A, f}$ is given by $(x, g) \mapsto\left(\sigma_{A} x, g+f(x)\right)$. A skew product has a natural $G$ action by translation on the $G$ coordinate. Two skew products into $G$ are isomorphic if they are topologically conjugate by a homeomorphism which intertwines the $G$ actions.

Question 31.1 (Bill Parry, the 90's). Can there exist an irreducible SFT $\sigma_{A}$ and an infinite family of pairwise nonisomorphic skew products constructed from skewing functions $f$ as above, where all the zeta functions $\zeta_{f}$ are the same?

Parry by example showed that finitely many nonisomorphic skew products over an irreducible SFT could share the same zeta function $\zeta_{f}$, but hoped the answer to Question 31.1 would be "no". He also showed the isomorphism of the skew products could be posed as a question of strong shift equivalence over $\mathbb{Z}_{+} G$ of defining matrices of the form $B$ above; for this see [23, 47]. For related work (in which the skewing function $f$ is Hölder into the real numbers), see [164].

\section{Classification OF SOFiC Shifts}

A sofic shift is a subshift which is a continuous quotient of a shift of finite type. The sofic shifts are in some ways the most natural finitely defined class of subshifts. For example, a subshift is sofic if and only if its language of allowed words is a regular language; and a subshift quotient of a sofic shift is again sofic (in contrast to the SFT case).

Problem 32.1. Classify sofic shifts up to topological conjugacy.

It is impossible to solve Problem 32.1 without first classifying SFTs - that problem comes first. Still, looking ahead, we'll sketch the state of the art for Problem 32.1 . 
Given a sofic shift $T$, the futures cover of $S$ (also called the future cover or Krieger cover $[127,119])$ is a one-block right-resolving block code $\pi_{\text {fut }}: S \rightarrow T$ from an (edge) SFT $S$ onto $T$, which can be defined by a labeling of a directed graph as follows. The vertices of the graph are the (finitely many) sets

$$
[x]=\{z[1, \infty): x(-\infty, 0] z[1, \infty) \in S\}
$$

defined from points $x$ of $T$. (Heuristically, $[x]$ represents the "possible futures" seen at time zero from the history of a point $x$.) There is an edge in the graph from $[x]$ to $[y]$ labeled $a$ if and only if there exists some sequence $a z_{1} z_{2} z_{3} \ldots$ in $[x]$ such that $z_{1} z_{2} z_{3} \ldots$ is in $[y]$. A version of this cover was first introduced, for irreducible sofic shifts, by R. Fischer; the construction is closely related to the classical "subset construction" of a minimal deterministic finite automaton in computer science (see [127]).

A key advance in the theory of sofic shifts was Krieger's insight [123] that the map $\pi_{\text {fut }}$ is canonical, in the following sense: if $\phi: T \rightarrow T^{\prime}$ is a topological conjugacy of two sofic shifts, with future covers $\pi_{\text {fut }}: S \rightarrow T$ and $\pi_{\text {fut }}^{\prime}: S^{\prime} \rightarrow T^{\prime}$, then there is a unique topological conjugacy $\widetilde{\phi}$ such that $\pi_{\text {fut }}^{\prime} \widetilde{\phi}=\pi_{\text {fut }} \phi$. So, the classification of the sofic shifts up to topological conjugacy amounts to the classification of a certain class of maps from SFTs up to topological conjugacy (we say two maps $\pi, \pi^{\prime}$ are topologically conjugate if there exist topological conjugacies $\phi, \widetilde{\phi}$ of their domain and range such that $\left.\pi^{\prime} \widetilde{\phi}=\phi \pi\right)$. This allows a rich collection of fine invariants [38], but further it leads to an algebraic classification approach, as follows.

The future covers, presented by labeled graphs, may equivalently be presented by certain matrices (" $\lambda$-matrices"). For example, a matrix $A=\left(\begin{array}{cc}a & b \\ b+c & 0\end{array}\right)$ describes a certain edge labeling of the directed graph with adjacency matrix $B=\left(\begin{array}{ll}1 & 1 \\ 2 & 0\end{array}\right)$, and this describes a one-block code from the edge SFT $\sigma_{B}$ onto a sofic shift whose alphabet is $\{a, b, c\}$. Formally, this $\lambda$-matrix has entries in the integral semigroup ring of the free group on symbols $a, b, c$.

Nasu [147] worked out an appropriate notion of strong shift equivalence for $\lambda$ matrices, as a part of a theory of bipartite codes for general subshifts, generalizing Williams' strong shift equivalence ideas. Say a symbolic monomial is a formal product over noncommuting variables; so, the entries of a $\lambda$-matrix are nonnegative integral combinations of symbolic monomials. Define an equivalence $\approx$ on $\lambda$-matrices by declaring $M \approx N$ if $M$ equals $N$ modulo a bijection of their underlying symbolic monomials. For example,

$$
\left(\begin{array}{cc}
0 & b \\
b+c & 2 a
\end{array}\right) \approx\left(\begin{array}{cc}
0 & a \\
a+d & 2 e
\end{array}\right) \approx\left(\begin{array}{cc}
0 & b b \\
b b+b c & 2 c b
\end{array}\right) .
$$

Now an elementary strong shift equivalence of $\lambda$-matrices $A, B$ is a pair of $\lambda$ matrices $U, V$ such that $A \approx U V$ and $B \approx V U$. Two $\lambda$-matrices are strong shift equivalent if they are related by a chain of elementary strong shift equivalences. Nasu showed strong shift equivalent $\lambda$-matrices define topologically conjugate quotient maps, and with Hamachi [87] he showed the converse.

One can reformulate this setup more algebraically [38]. Given a semigroup $\mathcal{S}$, let $\mathbb{Z}_{+} \mathcal{S}$ denote its integral semigroup semiring: the set of formal nonnegative integral combinations of elements of $S$, with addition and multiplication defined in the natural way, $\left(\sum_{i} m_{i} s_{i}\right)\left(\sum_{j} m_{j} s_{j}\right)=\sum_{i, j} m_{i} n_{j} s_{i} s_{j}$. If the semigroup contains 
a zero element (e.g., in a matrix semigroup, the zero matrix), then the reduced integral semigroup semiring $\mathcal{R}_{0}(S)$ is $\mathbb{Z}_{+} \mathcal{S}$ modulo that zero element.

Now, identify any finite matrix $A$ with the $\mathbb{N} \times \mathbb{N}$ matrix whose upper left corner is $A$ and whose other entries are zero. Regard a $\lambda$-matrix as a formal sum of integer matrices, e.g. replace

$$
\left(\begin{array}{cc}
0 & b \\
b+c & 2 a
\end{array}\right)=2\left(\begin{array}{ll}
0 & 0 \\
0 & a
\end{array}\right)+\left(\begin{array}{ll}
0 & b \\
b & 0
\end{array}\right)+\left(\begin{array}{ll}
0 & 0 \\
c & 0
\end{array}\right)
$$

with simply

$$
2\left(\begin{array}{ll}
0 & 0 \\
0 & 1
\end{array}\right)+\left(\begin{array}{ll}
0 & 1 \\
1 & 0
\end{array}\right)+\left(\begin{array}{ll}
0 & 0 \\
1 & 0
\end{array}\right)
$$

where we view this expression as an element of $\mathcal{R}_{0}(\mathcal{M})$, where $\mathcal{M}$ is the semigroup under multiplication of $\mathbb{N} \times \mathbb{N}$ matrices over $\mathbb{Z}_{+}$.

Williams' shift equivalence equations give a natural notion of shift equivalence of $\lambda$-matrices. The $\lambda$-matrices of future covers are shift equivalent if and only if their sofic shifts are eventually conjugate (i.e. all large powers are conjugate) [38]. Kim and Roush, generalizing their result for matrices over $\mathbb{Z}_{+}[102]$, produced an algorithm for deciding whether the $\lambda$-matrices of future covers are shift equivalent [104]. A key point of this work is the following. Let $T_{n}$ denote the semigroup of zeroone matrices with row sums at most 1 . Then elements of $\mathcal{R}_{0}\left(T_{n}\right)$ are shift equivalent in $\mathcal{R}_{0}(M)$ if and only if they are shift equivalent in the additively finitely generated ring $\mathcal{R}_{0}\left(T_{n}\right)$; and two given futures covers can always for some $n$ be presented as elements of $\mathcal{R}_{0}\left(T_{n}\right)$ [102].

In sum, at the level of decidability, the classification problems for SFTs and sofic shifts are at the same stage: eventual conjugacy is algebraic and decidable, while conjugacy remains mysterious. For some classes of sofic shift, the classification problem reduces explicitly to the classification of SFTs and the problem of extending conjugacies of subsystems to automorphisms [38, 39]. For mixing SFTs, after $[39,114,115,116]$, this extension problem reduces to the problem of knowing the dimension representation on the automorphism group of a mixing SFT.

Finally we mention it is possible to develop a dimension group approach to shift equivalence of sofic shifts [104, 38].

\section{Classification and flow Equivalence of General Shifts}

Matsumoto has developed a theory of shift equivalence and strong shift equivalence for arbitrary subshifts, vastly generalizing the work of Williams and Nasu. Nasu's defining $\lambda$-matrix is replaced by a Bratteli diagram with an additional structure, and the defining equations of shift and strong shift equivalence have analogues. Matsumoto also [139] associated to a subshift $\Lambda$ a $C^{*}$-algebra $\mathcal{O}_{\Lambda}$, generalizing the seminal construction of Cuntz and Krieger [61], and these $C^{*}$-algebras are stably isomorphic in the case that the underlying subshifts are flow equivalent. Thus, the flow equivalence of subshifts has a particular interest from the $C^{*}$ perspective. Matsumoto also developed flow equivalence invariants generalizing the Bowen-Franks group.

See [140] for a clear development of Matsumoto's theory at the level of topological dynamics independent of the $C^{*}$-algebras. For recent new entropylike invariants 
for subshifts, growing out of the theory, see $[124,143]$. For the $C^{*}$-algebras and associated ordered groups, see $[55,56,57,67,139,142]$ and their references. Below we will only define the most central invariants, and state two problems of Matsumoto.

First we consider Matsumoto's construction which associates a certain Bratteli diagram to a subshift. Say two points $x, y$ of a subshift $S$ are $k$-past equivalent $\left(x \sim_{k} y\right)$ if for every word $w$ of length $k$, the sequence $w x[k, \infty)$ occurs in $S$ (i.e. is the right half of a point of $S$ ) if and only if $w y[k, \infty)$ occurs in $S$. Given $x$ in $S$, set

$$
v_{k}(x)=\left\{y[k, \infty): y \in S, y \sim_{k} x\right\} .
$$

The finite vertex set $\mathcal{V}_{k}$ for level $k$ of the Bratteli diagram is $\left\{v_{k}(x): x \in S\right\}$. There is an edge labelled $a$ from $v_{k}(x)$ to $v_{k+1}(y)$ iff the sequence $a y[k+1, \infty)$ is in $v_{k}(x)$. A Bratteli diagram yields a set of sequences $x_{0} x_{1} x_{2} \ldots$ corresponding to labelings of infinite paths from $\mathcal{V}_{0}$; here, these are exactly the sequences $x[0, \infty)$ such that $x \in S$. The labeled edges from $\mathcal{V}_{k}$ to $\mathcal{V}_{k+1}$ are encoded in a $\lambda$-matrix $\mathcal{M}_{k, k+1}$, giving a sequence $\mathcal{M}=\left(\mathcal{M}_{k, k+1}\right)_{k \in \mathbb{Z}_{+}}$. We also have for $k>0$ a surjection $\mathcal{V}_{k+1} \rightarrow \mathcal{V}_{k}$ defined by the rule $v_{k}(x) \mapsto v_{k-1}(x)$. Let $I_{k, k+1}$ denote the $\left|\mathcal{V}_{k}\right| \times\left|\mathcal{V}_{k+1}\right|$ zero-one matrix describing this function, and $\mathcal{I}=\left(I_{k, k+1}\right)_{k}$ the corresonding sequence of matrices. Matsumoto pointed out that the matrices satisfy a fundamental commutation relation,

$$
\mathcal{I}_{k, k+1} \mathcal{M}_{k+1, k+2}=\mathcal{M}_{k, k+1} \mathcal{I}_{k+1, k+2}, \quad k \in \mathbb{Z}_{+} .
$$

Matsumoto defined a symbolic matrix system to be any pair $\mathcal{I}, \mathcal{M}$ such that $\mathcal{I}=$ $\left(I_{k, k+1}\right)_{k}$ is a sequence of zero-one matrices with nonzero row sums and all column sums $1 ; \mathcal{M}=\left(\mathcal{M}_{k, k+1}\right)_{k \in \mathbb{Z}_{+}}$is a sequence of $\lambda$ matrices with all letters coming from some finite alphabet; and the commutation relation (33.1) holds. (This implicitly assumes the matrix sizes are compatible with the multiplications.) A symbolic matrix system determines a Bratteli diagram whose set of label sequences, as above, equals $\{x[0, \infty): x \in S\}$ for some subshift $S$. The pair $(\mathcal{M}, \mathcal{I})$ arising from the explicit construction above using the $v_{k}(x)$ is the canonical symbolic matrix system of the subshift.

Two symbolic matrix systems $(\mathcal{M}, \mathcal{I})$ and $\left(\mathcal{M}^{\prime}, \mathcal{I}^{\prime}\right)$ are 1 -step strong shift equivalent over alphabets $\Sigma, \Sigma^{\prime}$ if there are sequences $\mathcal{H}_{k}, \mathcal{K}_{k}$ of $\lambda$-matrices, over alphabets $C, D$ respectively, such that the following hold for all $k>0$ :

$$
\begin{aligned}
\mathcal{I}_{k-1, k} \mathcal{M}_{k, k+1} & \approx \mathcal{H}_{k} \mathcal{K}_{k+1} & \mathcal{I}_{k-1, k}^{\prime} \mathcal{M}_{k, k+1}^{\prime} & \approx \mathcal{K}_{k} \mathcal{H}_{k+1} \\
\mathcal{H}_{k} \mathcal{I}_{k, k+1}^{\prime} & =\mathcal{I}_{k-1, k} \mathcal{H}_{k+1}, & \mathcal{K}_{k} \mathcal{I}_{k, k+1}^{\prime} & =\mathcal{I}_{k-1, k}^{\prime} \mathcal{K}_{k+1} .
\end{aligned}
$$

Let $B, C, D$ and $E$ be the alphabets used for the $\lambda$ matrices of $\mathcal{M}, \mathcal{H}, \mathcal{K}$ and $\mathcal{M}^{\prime}$. The meaning of the upper left $\approx$ above is that there is an injection $\phi: B \rightarrow C D$, $b \mapsto c d$, such that for every $k$, the map $\phi$ applied entrywise sends $\mathcal{I}_{k-1, k} \mathcal{M}_{k, k+1}$ to $\mathcal{H}_{k} \mathcal{K}_{k+1}$. The right side $\approx$ is defined in the same way with respect to some map $E \rightarrow D C$.

Finally, two symbolic matrix systems are strong shift equivalent if they are connected by a chain of 1-step strong shift equivalences of symbolic matrix systems.

Matsumoto showed that two subshifts are topologically conjugate if and only if their canonical symbolic matrix systems are strong shift equivalent [140, Theorem A]. He defined the natural notion of shift equivalence, and showed that two subshifts are eventually conjugate if their canonical symbolic matrix systems are shift equivalent. (In contrast to the SFT case, it appears to be open as to whether the 
canonical symbolic matrix systems of eventually conjugate subshifts must be shift equivalent, and it would be interesting to resolve this question.)

Lastly, suppose $\mathcal{M}, \mathcal{I}$ is a symbolic matrix system. Let $M, I$ be the pair of sequences of matrices over $\mathbb{Z}_{+}$which is the image of $\mathcal{M}, \mathcal{I}$ under the (augmentation) homomorphism which replaces each letter in the alphabet with 1 . (In the case that $M, I$ is the image of the canonical symbolic matrix system of a subshift, we will call $M, I$ the canonical matrix system of the subshift.) Then the sequence $I$ defines a dimension group $\mathbb{Z}_{I}$, as the following direct limit group:

$$
\mathbb{Z}^{\left|\mathcal{V}_{0}\right|} \stackrel{I_{0,1}}{\longrightarrow} \mathbb{Z}^{\left|\mathcal{V}_{1}\right|} \stackrel{I_{1,2}}{\longrightarrow} \mathbb{Z}^{\left|\mathcal{V}_{2}\right|} \stackrel{I_{2,3}}{\longrightarrow} \cdots \quad .
$$

The sequence $M$ defines an endomorphism $\lambda$ on $\mathbb{Z}_{I}$ :

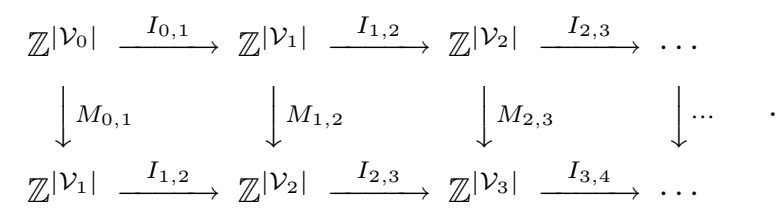

(Caveat: matrices appear in [140] with transposes; we have described the action as on row vectors to simplify notation.) The groups $K_{0}(M, I)$ and $K_{1}(M, I)$ are respectively the cokernel and kernel of the homomorphism Id $-\lambda: \mathbb{Z}_{I} \rightarrow Z_{I}[140$, Proposition 9.2]. These are generalizations of the Bowen-Franks group of an SFT (a nearly complete invariant of flow equivalence for irreducible SFTs [127, Sec. 13.6]). Also, they occur as $K$ groups of the $C^{*}$-algebras $\mathcal{O}_{\Lambda}$ associated to the subshift by Matsumoto; when $(M, I)$ is the canonical matrix system of the subshift (see [57]),

$$
K_{i}(M, I)=K_{i}\left(\mathcal{O}_{\Lambda}\right), \quad i=0,1 .
$$

Given a certain irreducibility condition on the subshift $\Lambda$, "aperiodic in past equivalence" (satisfied for example by the $\beta$-shifts [57]), the $C^{*}$-algebras $\mathcal{O}_{\Lambda}$ are purely infinite simple nuclear $C^{*}$-algebras ([142, Proposition 5.6], [141, Cor. 6.11], [57, Prop. 2.6, Thm. 3.4]). It is a consequence of the Kirchberg-Phillips-Rørdom theory (see [170]) that within the class of purely infinite simple nuclear $C^{*}$-algebras, the pair $K_{0}\left(\mathcal{O}_{\Lambda}\right), K_{1}\left(\mathcal{O}_{\Lambda}\right)$ is a complete invariant of stable isomorphism. For general subshifts $\Lambda$, the study of the $\mathcal{O}_{\Lambda}$ and related $C^{*}$-algebras is more subtle (see [57]); in particular the $K$ groups can carry a meaningful order ideal structure.

Problem 33.2. (Matsumoto) Which pairs of abelian groups occur in subshifts as $K_{0}(M, I), K_{1}(M, I)$ for some canonical matrix system $(M, I)$ ?

Problem 33.3. (Matsumoto) Classify subshifts up to flow equivalence.

\section{The Pisot Conjecture}

Intersecting symbolic dynamics is the huge field of substitutions, tilings and related topics, which merits its own problems survey. We'll state just one popular problem from this area: the so-called Pisot Conjecture.

A substitution rule is a map $\tau$ from some finite set $\mathcal{A}=\left\{a_{1}, \ldots a_{n}\right\}$ into words on $\mathcal{A}$, say $\tau: a_{i} \mapsto a_{i, 1} \cdots a_{i, m(i)}$. (The elements of $\mathcal{A}$ might be geometric shapes, with $\tau\left(a_{i}\right)$ corresponding to a tiling of a dilation of $a_{i}$.) There is an associated $n \times n$ matrix $A$ over $\mathbb{Z}_{+}$, given by $A(i, j)=\operatorname{card}\left\{k: a_{i, k}=a_{j}\right\}$. The substitution is irreducible Pisot if the characteristic polyonomial of $A$ is irreducible with a single root greater than 1 and every other root having modulus strictly less than 1 . 
The substitution rule iterates: inductively, $\tau^{n}\left(a_{i}\right)=\tau^{n-1}\left(a_{i, 1}\right) \cdots \tau^{n-1}\left(a_{i, m(i)}\right)$. A familiar construction associates to a substitution rule a substitutive shift $\sigma$ : the domain of this subshift is the set of the two sided sequences $x$ such that for all $n$, the word $x[-n, n]$ occurs in some $\tau^{k}\left(a_{i}\right)$ (when the nonnegative matrix $A$ is irreducible, this condition does not depend on the choice of $a_{i}$ ). When the matrix $A$ is primitive (e.g. when the substitution is irreducible Pisot), this subshift is uniquely ergodic.

Conjecture 34.1 (Pisot Conjecture). For an irreducible Pisot substitution, the associated substitutive shift has pure discrete spectrum.

The Pisot Conjecture has roots at least as far back as $[18,168]$. It relates to the work of many people, and has various equivalent formulations, especially in terms of tilings. See [14, 11], and their many references. For general background and problems on substitutions, see [167, 77].

\section{Nivat's Conjecture}

In this section, $\mathcal{A}$ denotes an arbitrary nonempty finite set.

Given a point $x$ in a doubly infinite sequence on $\mathcal{A}$, let $p_{x}(n)$ be the number of distinct words in $x$ of length $n$. M. Morse and G. A. Hedlund [144] proved that $x$ is a periodic sequence if and only if there exists $n$ in $\mathbb{N}$ such that $p_{x}(n) \leq n$.

There have been various approaches to generalizing this result, including the following conjecture of Nivat [155], in which $N_{x}\left(n_{1}, n_{2}\right)$ denotes the number of distinct $n_{1} \times n_{2}$ words occuring in $x$.

Conjecture 35.1 (Nivat). Suppose $x \in \mathcal{A}^{\mathbb{Z}^{2}}$. If there exist positive integers $n_{1}, n_{2}$ such that $N_{x}\left(n_{1}, n_{2}\right) \leq n_{1} n_{2}$, then $x$ is periodic.

Quas and Zamboni [166] proved a weaker statement of the conjecture, with the bound $n_{1} n_{2}$ replaced by $(1 / 16) n_{1} n_{2}$. The conjecture would be false if $n_{1} n_{2}$ were replaced by the bound $n_{1} n_{2}+1$; the natural generalization of the conjecture to $\mathbb{Z}^{3}$ is false; and there are periodic $x$ in $\mathcal{A}^{\mathbb{Z}^{2}}$ such that $N_{x}\left(n_{1}, n_{2}\right)>n_{1} n_{2}$ for all positive integer pairs $\left(n_{1}, n_{2}\right)$.

For more see [166], its references and its citers.

\section{Appendix A. Commuting SFTs And PeRiodic POINTS}

If bijections $S, T$ commute, then for each $n, S$ maps points of $T$-period $n$ to other points of $T$-period $n$. If $T$ has only $k$ points of period $n$, then these are periodic points of $S$, of $S$-period at most $k$.

Low-order periodic point conditions of this sort sometimes imply two maps cannot commute. For example, if $|\operatorname{Fix}(S)|=1$ and $|\operatorname{Fix}(T)|=0$, then $S$ and $T$ cannot commute, because the fixed point set of $S$ (one point) would have to be fixed by $T$ - but $T$ has no fixed point.

Here is a more interesting example.

Proposition A.1. $\sigma_{[2]}$ and $\sigma_{[3]}$ (the full shifts on two and three symbols) cannot commute.

Proof. Suppose $S$ is topologically conjugate to $\sigma_{[2]}, T$ is topologically conjugate to $\sigma_{[3]}$ and $S T=T S$. We make two claims:

(1) The two fixed points of $S$ are a $T$-orbit of size 2 .

(2) The single $S$-orbit of size 2 is a $T$-orbit of size 2 . 
To prove (1), note $S$ has two fixed points. Either they are fixed points of $T$, or they are a $T$-orbit of size 2 . If they are fixed by $T$, then there is just one more fixed point of $T$, which must then be fixed by $S$, so $S$ has a third fixed point. This is a contradiction. The proof of $(2)$ is very similar.

We now know the points of $S$ period 1 and 2 form two $T$-orbits of size 2 . There are exactly three $T$-orbits of size 2 . The remaining orbit is then an $S$-invariant set of two points, i.e. it contains points of $S$-period 1 or 2 . This is a contradiction.

To show that there is no periodic point obstruction to Conjecture 13.1, we prove a lemma. Given $U$, we let $\mathcal{O}_{n}(U)$ denote the set of $U$-orbits of size (cardinality) $n$, and we let $P_{n}^{o}(U)$ denote the points of least $U$-period $n$ (i.e., the union of the orbits $\left.\mathcal{O}_{n}(U)\right)$.

Lemma A.2. Suppose $S, T$ are bijections of countable sets $X, Y$ with discrete topology, $M \geq 2$ and the following hold:

(1) for all $n$, the cardinalities $a_{n}=\left|\mathcal{O}_{n}(S)\right|$ and $b_{n}=\left|\mathcal{O}_{n}(T)\right|$ are finite;

(2) with $d_{n}=a_{n}-b_{n}$, it holds that $d_{1} \geq M$ and $d_{n+1}-d_{n} \geq 3$ for $n \geq 1$;

(3) for all $n \geq M, T$ has at least one orbit of size $n$.

Then $S$ can commute with $T$.

Proof. We will define injections $f_{k}: P_{k}^{o}(S) \rightarrow Y$ such that $f_{k} S=T f_{k}$ and $Y$ is the disjoint union of the images of the $f_{k}$. Then $\cup_{k} f_{k}$ will define a conjugacy $f$ of $S$ to an automorphism of $T$.

We set $d_{0}=0, e_{0}=0, Y_{0}=Y, T_{0}=T$ and let $f_{0}$ be empty. Recursively, having defined a subsystem $Y_{k-1}$ of $Y$, the restriction $T_{k-1}$ of $T$ to $Y_{k-1}$, a number $e_{k-1}$, and the map $f_{k-1}$, we will define $f_{k}$ and $e_{k}$. (Then we define $Y_{k}$ as the complement in $Y_{k-1}$ of the image of $f_{k}$, and $T_{k}$ as the restriction of $T$ to $Y_{k}$.) The map $f_{k}$ is defined in two steps. First, pick $\left|\mathcal{O}_{k}\left(T_{k-1}\right)\right| S$-orbits of size $k$, and send their union bijectively to $P_{k}^{o}\left(T_{k-1}\right)$; this is possible because

$$
\left.\left|P_{k}^{o}(S)\right|-\left|P_{k}^{o}\left(T_{k-1}\right)\right| \geq\left|P_{k}^{o}(S)\right|-\mid P_{k}^{o}(T)\right) \mid \geq k d_{k} \geq k\left(d_{k-1}+3\right)>k>0 .
$$

Second, we define $e_{k}=\left|P_{k}^{o}(S)\right|-\left|P_{k}^{o}\left(T_{k-1}\right)\right|$, noting $e_{k}>k$, and we map the remaining $e_{k}$ points of $\left|P_{k}^{o}(S)\right|$ bijectively to a single $T_{k-1}$ orbit of size $e_{k}$. Subject to these constraints, $f_{k}$ is defined in such a way that $f_{k} S=T f_{k}$.

Clearly this process will produce the required bijection $f$, if at each stage $k$ there exists a $T_{k-1}$ orbit of size $e_{k}$. We have $e_{1}>M$, so this holds at $k=1$. For the rest, by hypothesis (2), it suffices to prove by induction on $k$ the claim that the finite sequence $e_{1}, \ldots, e_{k}$ is strictly increasing. So suppose $k>1$ and the claim holds at $k-1$. Then

$$
\left|\mathcal{O}_{k}(T)\right| \geq\left|\mathcal{O}_{k}\left(T_{k-1}\right)\right| \geq\left|\mathcal{O}_{k}(T)\right|-1
$$

and therefore

$$
\begin{aligned}
e_{k}-e_{k-1} & =k\left(\left|\mathcal{O}_{k}(S)\right|-\left|\mathcal{O}_{k}\left(T_{k-1}\right)\right|\right)-(k-1)\left(\left|\mathcal{O}_{k-1}(S)\right|-\left|\mathcal{O}_{k-1}\left(T_{k-2}\right)\right|\right) \\
& \geq k\left(\left|\mathcal{O}_{k}(S)\right|-\left|\mathcal{O}_{k}(T)\right|\right)-(k-1)\left(\left|\mathcal{O}_{k-1}(S)\right|-\left(\left|\mathcal{O}_{k-1}(T)\right|-1\right)\right) \\
& =k d_{k}-(k-1)\left(d_{k-1}+1\right)=k\left(d_{k}-d_{k-1}\right)+d_{k-1}-1>0
\end{aligned}
$$


Proposition A.3. Suppose $\sigma_{A}$ and $\sigma_{B}$ are mixing SFTs. Let $S$ and $T$ be their restrictions to the periodic points, with discrete topologies. Then for all large $n, S^{n}$ and $T$ can commute.

Proof. It is a straightforward exercise to use the eigenvalue structure of $A$ and $B$ to show that for all large $n$, the maps $S^{n}$ and $T$ satisfy the assumptions of Lemma A.2.

\section{Appendix B. Commuting SFTs from commuting matrices, Following NASU}

Note: $A B=B A$ does not guarantee that $\sigma_{A}, \sigma_{B}$ can commute (e.g., $[A]=$ $2, B=[3]$ in Prop. A.1). However, we have the following result of Nasu, which is given as a result on LR textile systems [150, Theorem 6.3(3)].

Theorem B.1. [150] Suppose $A, B$ are commuting $\mathbb{Z}_{+}$matrices. Then there is a compact metric space $W$ with selfhomeomorphisms $S, T$ such that

(1) $S T=T S$, and

(2) $S^{i} T^{j}$ is topologically conjugate to the SFT $\sigma_{A^{i} B^{j}}$ whenever $i>0$ and $j>0$.

Proof. We will recapitulate Nasu's construction. Suppose $A$ and $B$ are $n \times n$ matrices over $\mathbb{Z}_{+}$, with $A B=B A$. View $A$ and $B$ as adjacency matrices for two directed graphs, with disjoint edge sets and a common vertex set $\{1,2, \ldots n\}$. Say e.g. an $a b$ path from $i$ to $j$ is an $A$ edge from $i$ to some $k$ followed by a $B$ edge from that $k$ to $j$. " $A B=B A$ " means that for each pair $i, j$ the number of $a b$ paths from $i$ to $j$ equals the number of $b a$ paths from $i$ to $j$. Thus we can build a set $\mathcal{W}$ of (Wang) tiles

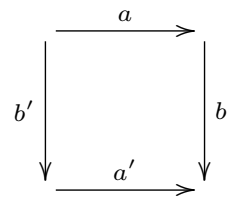

such that each $a b$ path is the top/right of exactly one tile and each $b a$ path is the left/bottom of exactly one tile. In the tile pictured, $a, a^{\prime}$ are $A$-edges and $b, b^{\prime}$ are $B$-edges. The tile pictured is then determined by either of the paths

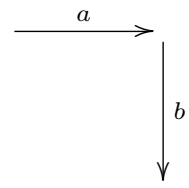

or

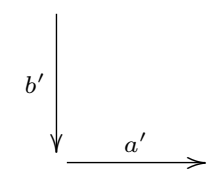

Let the tile sides be unit length and let $W$ be the space of infinite Wang tilings of the plane with $\mathcal{W}$, with tile corners on $\mathbb{Z}^{2}$. A tiling is a Wang tiling iff labels on adjoining tile sides match; here, the labels are edges. Formally, a point $w$ in $W$ is a function from $\mathbb{Z}^{2}$, where $w(\mathbf{n})$ is the Wang tile from the tiling which has lower left corner at coordinate $\mathbf{n}$. The space $W$ has the usual zero dimensional compact metrizable topology which is the relative topology of the product topology; concretely, we may define $\operatorname{dist}\left(w, w^{\prime}\right)=2^{-k}$ if $w(\mathbf{n})=w^{\prime}(\mathbf{n})$ for all $\|\mathbf{n}\|<k$ and $w(\mathbf{n}) \neq w^{\prime}(\mathbf{n})$ for some $\mathbf{n}$ with $\|\mathbf{n}\|<k$. For $\mathbf{v} \in \mathbb{Z}^{2}$, we let $\alpha^{\mathbf{v}}$ denote the shift 
map on $W$ in direction $\mathbf{v}$; that is, $\left(\alpha^{\mathbf{v}} w\right)(\mathbf{n})=w(\mathbf{n}+\mathbf{v})$. The shift maps $\alpha^{\mathbf{v}}$ are a commuting family of homeomorphisms.

Now let us examine a piece of a Wang tiling, below.

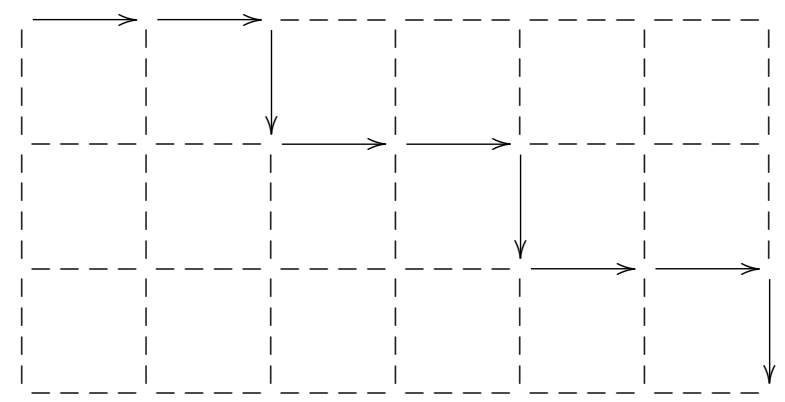

Following Nasu, we have horizontal coordinates increasing to the right and vertical coordinates increasing in the down direction (e.g., $(2,1)$ is two units to the right and one unit below $(0,0))$. Applying the bijections cited previously, tile by tile, we see that the solid lines of the figure determine the dashed lines. Considering this on the infinite tiles, we see the infinite "solid-line" path obtained by extending the solid line pattern of the figure determines the entire Wang tile. We conclude that $\alpha^{\mathbf{v}}$ is expansive for $\mathbf{v}=(2,1)$. Similarly, $\alpha^{\mathbf{v}}$ is expansive for $\mathbf{v}=(i, j)$ whenever $i>0$ and $j>0$.

To finish, we observe that for $i>0$ and $j>0$ we have $\alpha^{(i, j)}$ topologically conjugate to the edge SFT $X$ defined by the matrix $A^{i} B^{j}$. The correspondence $W \rightarrow X$ is obtained by projecting $w$ onto the infinite "solid-line" path described above, and viewing that infinite path as a point $x$ in $X$. For example, in the figure the solid line path would correspond to $x_{0} x_{1} x_{2}=\left(a_{0} a_{0}^{\prime} b_{0}\right)\left(a_{1} a_{1}^{\prime} b_{1}\right)\left(a_{2} a_{2}^{\prime} b_{2}\right)$ for some point $x$ in the SFT $\sigma_{A^{2} B}$, if we use symbols $a a^{\prime} b$ to denote edges in the graph whose adjacency matrix is $A^{2} B$.

The construction of Theorem B.1 works perfectly well (using Wang tilings of a quarter plane rather than the plane) to produce commuting onesided SFTs from commuting matrices. In the onesided setting, Nasu has a very striking converse (see [150, Theorem 3.13 and Prop. 6.1]): every $\mathbb{N}^{2}$ action generated by commuting onesided SFTs is topologically conjugate to an action given by the onesided version of the construction of Theorem B.1.

\section{Appendix C. LR Textile Systems}

Nasu's "textile systems" theory has proved to be a very useful tool for studying automorphisms and endomorphisms of SFTs. A reasonable exposition of this theory is beyond the scope of this article - we will not state various fundamental definitions and results - but we will try to give some indication of why the approach is natural and powerful.

Suppose that $T$ is an automorphism of a twosided edge SFT $S=\sigma_{A}$. Suppose that there exist Wang tiles just as in the construction of a $\mathbb{Z}^{2}$ action $\alpha$ on a space $W$ of Wang tilings as in the proof of Theorem B.1, where each $a b$ path is the top/right labeling of exactly one tile, and each $b a$ path is the left/bottom labeling of exactly one tile. Suppose also that each point $x=\ldots a_{-1} a_{0} a_{1} \ldots$ in the SFT $\sigma_{A}$ occurs 
as a horizontal bisequence inside some Wang tiling in $W$; that it then determines the rest of the Wang tiling; and finally that the horizontal edge bisequence directly below it in that tiling is the point $T(x)$. Given all this, the system of Wang tiles is said to define a 1-1 LR textile system, and $T$ is called an LR automorphism of $S$. In this case, the $\mathbb{Z}^{2}$ action generated by $\sigma_{A}$ and $T$ is conjugate to the $\mathbb{Z}^{2}$ shift action $\alpha$ on $W$, under the correspondence $\alpha^{(i, j)} \longleftrightarrow S^{i} T^{j}$.

Nasu shows [150, Theorem 6.29] that an automorphism $T$ of $S=\sigma_{A}$ is an LR automorphism if and only if it is a forward conjugacy (as we defined in Section 3 ). Using this, he easily deduces, given an automorphism $T$ of $\sigma_{A}$, that there is some $k \geq 0$ such that $S^{k} T$ is an LR automorphism of $S$ [150, p.66 and Theorem 6.29]. The pairs $S, T$ and $S, S^{k} T$ generate the same $\mathbb{Z}^{2}$ action, modulo change of coordinates, so the study of $\mathbb{Z}^{2}$ actions generated by an SFT and an automorphism of the SFT can be reduced to the study of the LR textile systems.

An important fact in Nasu's theory is that the matrix $B$ used (as in the proof of Theorem B.1) along with $A$ in the construction of the LR textile system is uniquely determined by $A$ and the automorphism $T$ [150, Corollary 7.25]. (We will call $B$ the accessory matrix for the LR automorphism $T$ of $\sigma_{A}$.) Moreover, for $i>0$ and $j \geq 0$, if $T$ is an LR automorphism of $\sigma_{A}$ with accessory matrix $B$, then $\left(\sigma_{A}\right)^{i} T^{j}$ is also an LR automorphism of $\sigma_{A}$, with accessory matrix $A^{i} B^{j}$.

Now suppose for this paragraph that $T$ is an LR automorphism of $\sigma_{A}, B$ is the accessory matrix for $T, A$ is $N \times N, i>0$ and $j \geq 0$. As in Theorem B.1, the map $\alpha^{(i, j)}$ is topologically conjugate to the edge SFT $\sigma_{A^{i} B^{j}}$. As in Sec. 2, we present the dimension module of $\sigma_{A}$ as $G_{A}=\left\{v \in V_{A}: \exists k>0, v A^{k} \in \mathbb{Z}^{n}\right\}$, where $V_{A}$ is the rational vector space of row vectors in the image of $A^{N}$. One can check for $i>0, j \geq 0$ that the action of $\alpha^{(i, j)}$ on $G_{A}$ is matrix multiplication by $A^{i} B^{j}$. If $i \geq N$, then $\operatorname{rank}\left(A^{i} B^{j}\right)=\operatorname{dim}\left(V_{A}\right)$, and the action of $T$ on the dimension module determines the matrix $A^{i} B^{j}$, which is the unique accessory matrix of the LR automorphism $\left(\sigma_{A}\right)^{i} T^{j}$ of $\sigma_{A}$. If $T^{\prime}$ is another LR autmorphism of $\sigma_{A}$ such that $T$ and $T^{\prime}$ have the same action on the dimension module, and $i \geq N$, it follows that the SFTs $\left(\sigma_{A}\right)^{i} T^{j}$ and $\left(\sigma_{A}\right)^{i} T^{j}$ must be topologically conjugate.

If $T$ is an automorphism of $\sigma_{A}$, let $k \geq 0$ be such that $\left(\sigma_{A}\right)^{k} T$ is an LR automorphism of $\sigma_{A}$. It follows from the discussion above that whenever $i \geq j k+N>0$, the topological conjugacy class of $\left(\sigma_{A}\right)^{i} T^{j}$ is determined by $(i, j)$ and the action of $T$ on the dimension module. (I do not know how to prove this without going through the textile systems.)

The same argument applies (perhaps for a smaller $k$ ) to the inverses of $T$ and $T^{\prime}$. This tells us that if two automorphisms $T, T^{\prime}$ of $S=\sigma_{A}$ have the same action on the dimension group, then for all integral vectors $(i, j)$ in some open cone around $(1,0)$, the map $S^{i} T^{j}$ is topologically conjugate to $S^{i}\left(T^{\prime}\right)^{j}$.

It is important to appreciate that the $\mathbb{Z}^{2}$ dynamics generated by $\sigma_{A}$ and $T$ are far from determined by this fine parametrization of the $\alpha^{(i, j)}$ dynamics in an open cone. For example, we could take $\sigma_{A}, T$ to be $\sigma_{A}, S$ in Example 13.2. Here $T$ acts like the identity on the dimension module, but the $\mathbb{Z}^{2}$ dynamics of $\sigma_{A}, T$ are quite different from those of $\sigma_{A}$, Id.

We want to indicate just a little of the further structure behind the LR systems. Nasu shows that if $T$ is an LR automorphism of an edge shift $S=\sigma_{A}$, then $T$ has memory 0 and $T^{-1}$ has anticipation 0 , and conversely if $T$ has memory 0 and $T^{-1}$ has anticipation 0 then $T^{[k]}$ is an LR automorphism of $S^{[k]}$ for some integer 
$k \geq 1$ [154, Prop. 5.1]. (Here $S^{[k]}$ denotes the $k$-block presentation edge SFT on sequences $\Sigma_{A^{[k]}}=\left\{x^{[k]}: x \in \Sigma_{A}\right\}$, where for $i$ in $\mathbb{Z}$ the $i$ th coordinate of $x^{[k]}$ is defined to be $\left(x^{[k]}\right)_{i}=\left(x_{i} \cdots x_{i+k-1}\right)$; and $T^{[k]}$ denotes the automorphism of $S^{[k]}$ induced by $T$, sending $x^{[k]}$ to $(T x)^{[k]}$.)

If $S, T$ are homeomorphisms generating a $\mathbb{Z}^{2}$ action conjugate to the action $\alpha$ on some LR textile system, under the correspondence $S^{i} T^{j} \longleftrightarrow \alpha^{(i, j)}$, then Nasu declares that $T$ is an ELR (essentially LR) automorphism of $S$. He shows that the following conditions are equivalent for an expansive automorphism $T$ of a SFT $\sigma_{A}$ (see [154], following [151]).

(1) $T$ is an ELR automorphism of $\sigma_{A}$.

(2) $T^{n}$ is an LR automorphism of $\sigma_{A}$ for some (all sufficiently large) $n \geq 1$.

(3) $T$ is in the expansive component of $\sigma_{A}$ (in the sense of [41]) for the $\mathbb{Z}^{2}$ action $\alpha$ given by $\alpha^{(i, j)}=\sigma_{A}^{i} T^{j}$.

The equivalence of (1) and (2) is a consequence of [150, Prop. 8.8 and Theorem $7.22(2)]$. Nasu has an example in which $T$ is a nonexpansive automorphism such that $T$ has memory $0, T^{-1}$ has anticipation 0 and for all $n \geq 1$ the automorphism $T^{n}$ is not LR [149] - although $T$ is ELR and $T^{[2]}$ is an LR automorphism of $S^{[2]}$ [150, Section 10, Example 2]. A nonexpansive ELR automorphism of an SFT $\sigma_{A}$ must lie on the boundary of an expansive component, because a nonexpansive LR automorphism must lie on the boundary of an expansive component, [150, Cor. $6.5(2)]$.

Lastly, we explain the "LR" terminology. The Wang tiles of the LR textile system can be regarded as edges in a directed graph; the initial vertex of the tile is its labelled left side, and the terminal vertex is its labeled right side. From a bisequence of tiles in the corresponding edge SFT, one can project onto the bisequence of top edges or onto the bisequence of bottom edges. These maps (when the textile system is LR) are respectively left resolving and right resolving maps between SFTs. (E.g., the left resolving property follows from the condition that for any pair of a horizontal edge $a$ and a vertical edge $b$ such that the tyerminal vertex of $a$ is the initial vertex of $b$, there exists exactly one Wang tile whose upper edge is $a$ and whose right edge is $b$.) The structure of resolving maps is fundamental to the results obtained so far with textile systems. For a relatively short introduction to textile systems, see [151]; for a thorough introduction, [150]; for the state of the art, [154].

\section{Appendix D. Commuting SFTs From matrices COMmuting on Dimension}

Let us verify Conjecture 13.1 in a special case (in fact proving a somewhat stronger result for that case).

Proposition D.1. Suppose $A, B$ are primitive integral matrices and there is an isomorphism of dimension groups $G_{A} \rightarrow G_{B}$ which carries the action of $A$ to an automorphism of $G_{B}$ commuting with the action of $B$. Then for all large $k$, the SFT $\left(\sigma_{A}\right)^{k}$ can commute with $\sigma_{B}$ (and thus with $\left(\sigma_{B}\right)^{j}$ for all $j>0$ ).

Proof. Let $A$ be $m \times m$ and let $B$ be $n \times n$. We use the notation and presentation of the dimension groups given as background in Section 2. E.g., $G_{A}=\left\{v \in V_{A}\right.$ : $\exists k>0$ such that $\left.v A^{k} \in \mathbb{Z}^{m}\right\}$, where $V_{A}=\left\{v A^{m}: v \in \mathbb{Q}^{m}\right\}$. The isomorphism $G_{A} \rightarrow G_{B}$ is the restriction to $G_{A}$ of a unique rational vector space isomorphism $\phi: V_{A} \rightarrow V_{B}$. Extend $\phi$ to a linear transformation $\phi^{\prime}: \mathbb{Q}^{m} \rightarrow \mathbb{Q}^{n}$ by declaring 
$\phi$ to vanish on $\left\{v \in \mathbb{Q}^{m}: v A^{m}=0\right\}$, which is the $A$-invariant subspace of $\mathbb{Q}^{m}$ complementary to $V_{A}$. Let $E$ be the $m \times n$ matrix over $\mathbb{Q}$ such that $\phi^{\prime}(v)=v E$ for $v \in \mathbb{Q}^{m}$. Let $C$ be the $n \times n$ matrix such that $w C=0$ if $w B^{n}=0$ and $v E C=v A E$ for $v \in \mathbb{Q}^{m}$. Then $B C=C B$.

The map $v \mapsto v E$ defines an isomorphism $G_{A} \rightarrow G_{B}$ which conjugates the action of $A$ on $G_{A}$ to the action of $C$ on $G_{B}$. Because $B C=C B$, for any $j>0$ the map $v \mapsto v E B^{j}$ also conjugates the action of $A$ on $G_{A}$ to the action of $C$ on $G_{B}$. The rows of $E$ must lie in $G_{B}$; thus for any large enough $j$ the matrix $E B^{j}$ will have integer entries. Because $B$ is primitive, for large $j$ these entries will also be strictly postive. So after passing to suitable $E B^{j}$, we may assume the entries of $E$ are strictly positive integers.

For $1 \leq i \leq n$, let $e_{i}$ be a canonical basis vector in $\mathbb{Q}^{n}$ and let $w_{i}$ be the element of $G_{B}$ such that $w_{i} B^{n}=e_{i} B^{n}$. Let $v_{i}$ be the element of $G_{A}$ such that $v_{i} E=w_{i}$. For $k>0$,

$$
\begin{aligned}
e_{i} C^{k} & =\left[w_{i}+\left(e_{i}-w_{i}\right)\right] C^{k} \\
& =w_{i} C^{k} \quad \text { by definition of } C \\
& =v_{i} E C^{k}=v_{i} A^{k} E .
\end{aligned}
$$

For all large $k, v_{i} A^{k}$ has positive integer entries, and therefore so does $v_{i} A^{k} E$. We have shown that for all large $k$, the matrix $C^{k}$ is positive integral with $B C^{k}=C^{k} B$. As explained in Appendix B, this implies that the SFTs $\sigma_{B}$ and $\sigma_{C^{k}}$ can commute. It remains to show that for all large $k$, the SFTs $\sigma_{C^{k}}$ and $\sigma_{A^{k}}$ are topologically conjugate.

Let $D$ be the $n \times m$ matrix over $\mathbb{Q}$ such that $v E D=v$ if $v \in G_{A}$ and $w D=0$ if $w B^{n}=0$. We have then also that $w D E=w$ if $w \in G_{B}$. Given $k>0$, define the matrix $F=D A^{k}$. For $1 \leq i \leq n$,

$$
\begin{aligned}
e_{i} F & =\left[w_{i}+\left(e_{i}-w_{i}\right)\right] D A^{k} \\
& =w_{i} D A^{k} \quad \text { by definition of } D \\
& =v_{i} A^{k} .
\end{aligned}
$$

Therefore, for all large $k$ the matrix $F$ is, like $E$, a positive integral matrix. For $k \geq n$, we have $F E=D A^{k} E=D E C^{k}=C^{k}$, where the last equality holds because the matrices $D E C^{k}$ and $C^{k}$ have the same action on $V_{B}$ and also the same action (annihilation) on the complementary $B$-invariant subspace $\left\{w: w B^{n}=0\right\}$. Similarly, for $k \geq m$ we have $E F=E D A^{k}=A^{k}$.

Thus for large $k$ the pair $E, F$ gives a strong shift equivalence over $\mathbb{Z}_{+}$of $A^{k}$ and $C^{k}$. This establishes the required topological conjugacy.

\section{Appendix E. Primitive matrices SE But not SSE over $\mathbb{Z}_{+}[1 / p]$}

Suppose $\mathcal{R}$ is a unital subring of $\mathbb{R}$. If two primitive matrices are shift equivalent over $\mathcal{R}_{+}$, must they be strong shift equivalent over $\mathcal{R}_{+}$? For $\mathcal{R}=\mathbb{Z}$, Kim and Roush showed the answer is no; for $\mathcal{R}=\mathbb{Q}$, the question is open. The purpose of this section is to point out that there are nondiscrete $\mathcal{R}$ for which the answer is no; the question is not just a matter of whether $\mathcal{R}$ is dense in $\mathbb{R}$.

For their counterexample [112], Kim and Roush produced $7 \times 7$ primitive matrices $A$ and $B$ which are shift equivalent over $\mathbb{Z}_{+}$but are not strong shift equivalent over $\mathbb{Z}_{+}$. The characteristic polynomial of both matrices is $f(x)=x^{7}-23 x^{4}-28 x^{3}-$ 
$33 x^{2}-17 x+1$, which is irreducible. Let $F$ denote the algebraic number field $\mathbb{Q}[x] / f(x)$ and let $\mathcal{O}_{F}$ denote the algebraic integers of $F$.

Proposition E.1. Let $A, B, f, F$ be as above. Suppose $p$ is an odd rational prime and the ideal $(p)$ is prime in the number field $F$. Let $\mathcal{R}$ denote the ring $\mathbb{Z}[1 / p]$. Then the primitive matrices $A$ and $B$ are shift equivalent over $\mathcal{R}_{+}$, but they are not strong shift equivalent over $\mathcal{R}_{+}$.

Proof. The proof is to verify that the obstruction to SSE of $A$ and $B$ over $\mathbb{Z}_{+}$ demonstrated in [112] is also an obstruction to SSE of $A$ and $B$ over $\mathcal{R}_{+}$. We must assume familiarity with [112]. First, $\mathcal{R}$ is a principal ideal domain and therefore for $\Lambda=\mathcal{R}$ we still have the isomorphism $\pi_{1}(R S(\Lambda), A) \rightarrow \operatorname{Aut}\left(s_{A / \Lambda}\right)$. The only other item to check is that $\operatorname{sgc}_{2}$ vanishes on $\operatorname{Aut}\left(s_{A / \Lambda}\right)$.

Because $A$ is nonsingular, $\operatorname{Aut}\left(s_{A / \mathcal{R}}\right)$ can be identified with the group of nonsingular matrices $V$ over $\mathbb{Q}$ which commute with $A$ and for some $n>0$ satisfy that $V A^{n}$ and $V^{-1} A^{n}$ have all entries in $\mathcal{R}$. This forces $\operatorname{det} V$ to be some power of $p$; so, $V \in \operatorname{Aut}\left(s_{A / \mathcal{R}}\right)$ if and only if $V$ has entries in $\mathcal{R}$ and $\operatorname{det} V=p^{k}$ for some $k \in \mathbb{Z}$.

In [112], for $\Lambda=\mathbb{Z}$ Kim and Roush went on to identify $\operatorname{Aut}\left(s_{A / \Lambda}\right)$ with a subgroup of the units group $\mathbb{U}_{F}$ of $\mathcal{O}_{F} ;$ with $\Lambda=\mathcal{R}$, we similarly identify $\operatorname{Aut}\left(s_{A / \Lambda}\right)$ with a subgroup of the units of $\mathcal{O}_{F}[1 / p]$. Because $(p)$ is a prime ideal, a unit of $\mathcal{O}_{F}[1 / p]$ is an element of $\mathbb{U}_{F}$ multiplied by a power of $p$. Kim and Roush already showed that $\mathrm{sgc}_{2}$ vanishes on $\mathbb{U}_{F}$ so it only remains to verify that $\mathrm{sgc}_{2}$ vanishes on $p$. Translated back to matrices, we have to check that $\mathrm{sgc}_{2}$ vanishes on the sse over $\mathcal{R}$ given by $\left(D, A D^{-1}\right)$, where $D$ is the $7 \times 7$ diagonal matrix $p I$. The computation of $\mathrm{sgc}_{2}$ only depends on the matrix entries mod 4 , so this is the computation for $(I, A)$ or $(-I,-A)$, and it was already shown by Kim and Roush that $\mathrm{sgc}_{2}$ vanishes on these.

It remains to know that there are odd rational primes $p$ such that $(p)$ is a prime ideal in the number ring $\mathcal{O}_{F}$. For this I thank Larry Washington for the algebraic number theory to follow.

First, $(p)$ will be prime in $\mathcal{O}_{F}$ if $f(x)$ is irreducible modulo $p$ and $p$ does not divide the index $F=\mathcal{O}_{F} / \mathbb{Z}(x)$; this is a special case of a general result on the factorization of $(p)$ in $\mathcal{O}_{F}$ [58, Theorem 4.8.13]. Second, that index must divide the discriminant of the polynomial $f(x)$ [58, Proposition 4.4.4].

From the computer algebra program PARI, we find the prime factors of the discriminant of our polynomial $f$ are $2,3,47$ and 78901 . The command "factormod" in Pari reveals that $f$ is irreducible $\bmod p$ for $p=5,31,61,71 \ldots$ These give us the required examples.

Moreover, there are infinitely many such $p$. Because $f$ is irreducible with degree 7, 7 divides the order of the Galois group, which therefore contains an element $g$ of order 7 . The Chebotarev density theorem says that there are infinitely many prime ideals $\mathrm{P}$ in the Galois closure with mod P Galois group generated by g. Namely, the mod $\mathrm{P}$ Frobenius is $\mathrm{g}$. If $p$ is the rational prime over which $\mathrm{P}$ lies, then the mod $p$ polynomial must be irreducible.

\section{APPENDIX F. EXAMPLES FOR THE ONESIDED SOFIC CLASSIFICATION PROBLEM}

In this section, all shifts are onesided. The purpose is to give a class of examples which rule out certain approaches to the classification problem for one sided sofic shifts. 
Proposition F.1. For any $n$ there is a mixing SFT $\sigma_{A}$, and two labellings on the graph $G_{A}$ giving the canonical predecessor covers of topologically conjugate sofic shifts $S^{\prime}$ and $S^{\prime \prime}$, such that the following hold.

(1) The matrix $A$ is totally amalgamated (so, no labeling of a smaller graph can define a topologically conjugate factor map).

(2) Any automorphism of $\sigma_{A}$ which conjugates the covers must have coding length at least $n$.

Remark F.2. $S^{\prime}$ and $S^{\prime \prime}$ above are topologically conjugate if and only if those canonical predecessor covers are topologically conjugate.

Proof. Our sofic systems here will be near-Markov sofic shifts, i.e., quotients obtained by collapsing only finitely many points from a covering SFT.

Notation: we will describe labeled graphs using letters for labeled-graph adjacency matrices. E.g., the matrix $(a+b+c)$ describes the labeling of the three edges of the graph for the matrix (3) using symbols $a, b, c$.

We will describe two labelings of a graph $G=G_{A}$, giving quotient maps $p^{\prime}, p^{\prime \prime}$ onto sofic systems $T^{\prime}, T^{\prime \prime}$ and defining quotient relations $E^{\prime}, E^{\prime \prime}$ on $\sigma_{A}$. The sofic systems will be conjugate iff there is an automorphism $U$ of $\sigma_{A}$ sending $E^{\prime}$ to $E^{\prime \prime}$.

The adjacency matrix $A$ will have the block form $A=\left(\begin{array}{cc}W & X \\ Y & Z\end{array}\right)$ where $Z=(3)$;

$Y$ and $X$ are respectively the column and row vector with every entry equal to 1 ; and $W$ is totally amalgamated with all entries in $\{0,1\}$. The choices for $X, Y, Z$ guarantee $A$ is primitive and therefore $\sigma_{A}$ is mixing.

In both adjacency matrices for the labelings, $A^{\prime}$ and $A^{\prime \prime}$, the matrix $Z$ will be replaced by $(a+b+c)$. Certain entries of $W$ will also have labels from $a, b, c$. All other entries have distinct labels. The labelings with $a, b, c$ in $W$ will serve to identify certain periodic orbits of $S_{W}$ with orbits of $S_{Z}$, and there will be only finitely many such orbits identified. It follows from the structure theory of automorphisms of onesided SFTs [32] that any automorphism of the SFT $\sigma_{A}$ must act like the identity on the subsystem $\sigma_{W}$ (because $A$ is totally amalgamated, $W$ is zero-one, and the only automorphisms of the graph with adjacency matrix $A$ are the permutations of the three edges corresponding to $Z$ ). Thus there will be an automorphism $U$ of $\sigma_{A}$ sending $E^{\prime}$ to $E^{\prime \prime}$ iff there is an automorphism of $\sigma_{A}$ which moves orbits in $\sigma_{Z}$ in a way to line up the identifications.

Here is one way to go about this. Given $N$, enumerate the $\sigma_{Z}$ periodic orbits of period at most $N$ as $\mathcal{O}_{i}, 1 \leq i \leq I$. From the $i$ th orbit, pick a point $x_{i}$. Let $D$ be a $d \times d$ matrix which is the direct sum of cyclic periodic matrices $M_{i}, 1 \leq i \leq I$, with $M_{i} k \times k$ if $x_{i}$ has period $k$. Then $W$ will be $d \times d$ with $D$ as a submatrix. Make choices of 0 and 1 for the unspecified entries of $W$ so that all columns of $W$ are distinct (for example, for $1 \leq i<I$ allow a single transition from some row through $M_{i}$ to some row through $M_{i+1}$, and set all other entries equal to 0 ).

Now put labels on the entries of the $M_{i}$ which match $x_{i}$. E.g. if $x_{i}$ is the point $a b b a b b a b b \ldots$, then the three 1's in $M_{i}$ are replaced in order by $a, b, b$. Aside from the dictated labelings on $Z$ and the $M_{i}$, let all other labels be distinct. This defines the matrix $A^{\prime}$, and $E^{\prime}$ collapses only certain pairs of finite orbits, one pair for each $\mathcal{O}_{i}$.

Now let $V$ be a given automorphism of the 3 shift $\sigma_{Z}$. $A^{\prime \prime}$ will be defined in the same way, except that the labeling on $M_{i}$ will correspond not to $x_{i}$ but rather to $V\left(x_{i}\right)$. Again, an automorphism $U$ of $\sigma_{A}$ must act like the identity on $\sigma_{W}$. So, 
$U$ will send $E^{\prime \prime}$ to $E^{\prime}$ iff on $\sigma_{Z}$ it acts like $V$. The arbitrary automorphism $V$ of the three shift $\sigma_{Z}$ can be extended to an automorphism of $\sigma_{A}$ : any sequence of splittings and amalgamations for the matrix (3) can be extended to $A$.

There are infinitely many automorphisms of the three shift [32], and they are separated by their actions on periodic points. Given $n$, we can pick $V$ of minimal coding length at least $n$, and then pick $N$ such that none of the finite set of automorphisms of $\sigma_{Z}$ which have shorter coding length have the same action as $V$ on all periodic points of period less than or equal to $N$. For this $V$, with the construction above we obtain that the extension $U$ has minimal coding length at least $n$.

Further progress on Problem 21.2 may involve Ashley's decomposition theory for automorphisms of a onesided SFT [7].

\section{REFERENCES}

[1] R. Adler, L. Goodwyn and B. Weiss, Equivalence of topological Markov shifts, Israel J. Math. 27 (1977), no. 1, 48-63.

[2] R. Adler and B. Marcus, Topological entropy and equivalence of dynamical systems, Mem. Amer. Math. Soc.20 (1979), no. 219, iv+84 pp.

[3] J.-P. Allouche and J. Shallit, Automatic sequences. Theory, applications, generalizations, Cambridge University Press, Cambridge, 2003.

[4] V. de Angelis and S. Tuncel, Handelman's theorem on polynomials with positive multiples, in Codes, systems, and graphical models (Minneapolis, MN, 1999), 439-445, IMA Vol. Math. Appl. 123, Springer, New York, 2001.

[5] J. Ashley, Resolving factor maps for shifts of finite type with equal entropy, Ergodic Theory Dynam. Systems 11 (1991), no. 2, 219-240.

[6] J. Ashley, An extension theorem for closing maps of shifts of finite type, Trans. Amer. Math. Soc. 336 (1993), no. 1, 389-420.

[7] J. Ashley, Marker automorphisms of the one-sided d-shift, Ergodic Theory Dynam. Systems 10 (1990), no. 2, 247-262.

[8] J. Ashley, B. Marcus and S. Tuncel, The classification of one-sided Markov chains, Ergodic Theory Dynam. Systems 17 (1997), no. 2, 269-295.

[9] K. Baker, Strong shift equivalence of $2 \times 2$ matrices of nonnegative integers, Ergodic Theory Dynam. Systems 3 (1983), no. 4, 501-508.

[10] K. A. Baker, Strong shift equivalence and shear adjacency of nonnegative square integer matrices, Linear Algebra Appl. 93 (1987), 131-147.

[11] V. Baker, M. Barge and J. Kwapisz, Geometric realization and coincidence for reducible non-unimodular Pisot tiling spaces with an application to $\beta$-shifts, Numration, pavages, substitutions. Ann. Inst. Fourier (Grenoble) 56 (2006), no. 7, 2213-2248.

[12] J. Barth and A. Dykstra, Weak equivalence for shifts of finite type, preprint 2007.

[13] J. van den Berg and J.E. Steif, On the existence and nonexistence of finitary codings for a class of random fields, Ann. Probab. 27 (1999), no. 3, 1501-1522.

[14] V. Berth and A. Siegel, Tilings associated with beta-numeration and substitution, Integers 5 (2005), no. 3, A2, 46 pp. (electronic).

[15] F. Blanchard and A.Maass, Dynamical properties of expansive one-sided cellular automata, Israel J. Math. 99 (1997), 149-174.

[16] F. Blanchard, A. Maass and A. Nogueira, eds., Topics in symbolic dynamics and applications (Temuco, 1997), 57-88, London Math. Soc. Lecture Note Ser. 279, Cambridge Univ. Press, Cambridge, 2000.

[17] F. Blanchard and P. Tisseur, Some properties of cellular automata with equicontinuity points, Ann. Inst. H. Poincar Probab. Statist. 36 (2000), no. 5, 569-582.

[18] E. Bombieri and J. E. Taylor, Which distributions of matter diffract? An initial investigation, International workshop on aperiodic crystals (Les Houches, 1986), J. Physique 47 (1986), no. 7, Suppl. Colloq. C3, C3-19-C3-28.

[19] D. Boyd, The beta expansion for Salem numbers, Organic mathematics (Burnaby, BC, 1995), 117-131, CMS Conf. Proc., 20, Amer. Math. Soc., Providence, RI, 1997. 
[20] M. Boyle. Lower entropy factors of sofic systems, Ergodic Theory Dynam. Systems 3 (1983), no. 4, 541-557.

[21] M. Boyle, Symbolic Dynamics and Matrices, in Combinatorial and Graph-Theoretical Problems in Linear Algebra, IMA Volumes in Math. and its Applications 50 (1993), 1-38.

[22] M. Boyle, Factoring factor maps, J. London Math. Soc. (2) 57 (1998), no. 2, 491-502.

[23] M. Boyle, Positive K-theory and symbolic dynamics. Dynamics and randomness (Santiago, 2000), 31-52, Nonlinear Phenom. Complex Systems 7, Kluwer Acad. Publ., Dordrecht, 2002.

[24] M. Boyle, Some sofic shifts cannot commute with nonwandering shifts of finite type, Illinois J. Math. 48 (2004), no. 4, 1267-1277.

[25] M. Boyle, Putnam's resolving maps in dimension zero, Ergodic Theory Dynam. Systems 25 (2005), no. 5, 1485-1502.

[26] M. Boyle, J. Buzzi and R. Gómez, Almost isomorphism for countable state Markov shifts, J. Reine Angew. Math. 592 (2006), 23-47.

[27] M. Boyle and T. Downarowicz, The entropy theory of symbolic extensions, Inventiones Math. 156 (2004), 119-161.

[28] M. Boyle and T. Downarowicz, Symbolic extension entropy: $C^{r}$ examples, products and flows Discrete Contin. Dyn. Syst. 16 (2006), no. 2, 329-341.

[29] M. Boyle and U. Fiebig, The action of inert finite-order automorphisms on finite subsystems of the shift, Ergodic Theory Dynam. Systems 11 (1991), no. 3, 413-425.

[30] M. Boyle, D. Fiebig and U. Fiebig, A dimension group for local homeomorphisms and endomorphisms of onesided shifts of finite type, J. Reine Angew. Math. 487 (1997), $27-59$.

[31] M. Boyle, D. Fiebig and U. Fiebig, Residual entropy, conditional entropy and subshift covers, Forum Math. 14 (2002), 713-757.

[32] M. Boyle, J. Franks and B. Kitchens, Automorphisms of one-sided subshifts of finite type, Ergodic Theory Dynam. Systems 10 (1990), no. 3, 421-449.

[33] M. Boyle and D. Handelman, The spectra of nonnegative matrices via symbolic dynamics, Annals of Math. 133 (1991) 249-316.

[34] M. Boyle and D. Handelman, Algebraic shift equivalence and primitive matrices, Trans. Amer. Math. Soc. 336 (1993), no. 1, 121-149.

[35] M. Boyle and D. Handelman, Orbit equivalence, flow equivalence and ordered cohomology, Israel J. Math. 95 (1996), 169-210.

[36] M. Boyle and B. Kitchens, Periodic points for onto cellular automata, Indag. Math. (N.S.) 10 (1999), no. 4, 483-493.

[37] M. Boyle, B. Kitchens and B. Marcus, A note on minimal covers for sofic systems Proc. Amer. Math. Soc. 95 (1985), no. 3, 403-411.

[38] M. Boyle and W. Krieger, Almost Markov and shift equivalent sofic systems, Proceedings of Maryland Special Year in Dynamics 1986-87, Springer Lecture Notes in Math. 1342 (1988), 33-93, Springer, Berlin.

[39] M. Boyle and W. Krieger, Automorphisms and subsystems of the shift, J. Reine Angew. Math. 437 (1993), 13-28.

[40] M. Boyle and B. Lee, Jointly periodic points in cellular automata: computer exploraitons and conjectures, Experimental Math., to appear.

[41] M. Boyle and D. Lind, Expansive subdynamics, Trans. Amer. Math. Soc. 349 (1997), no. $1,55-102$.

[42] M. Boyle and D. Lind, Small polynomial matrix presentations of nonnegative matrices, Linear Algebra Appl. bf 355 (2002), 49-70.

[43] M. Boyle, D. Lind and D. Rudolph The automorphism group of a shift of finite type, Trans. Amer. Math. Soc. 306 (1988), no. 1, 71-114.

[44] M. Boyle and A. Maass, Expansive invertible onesided cellular automata, J. Math. Soc. Japan 52 (2000), no. 4, 725-740; Erratum, J. Math. Soc. Japan 56.

[45] M. Boyle, B. Marcus and P. Trow, Resolving maps and the dimension group for shifts of finite type, Mem. Amer. Math. Soc. 70 (1987), no. 377, vi+146 pp.

[46] M. Boyle and M. Schraudner, $Z^{d}$ group shifts and Bernoulli factors, Ergodic Theory Dynam. Systems, to appear.

[47] M. Boyle and M. Sullivan, Equivariant flow equivalence for shifts of finite type, by matrix equivalence over group rings, Proc. London Math. Soc. (3) 91 (2005), no. 1, 184-214. 
[48] M. Boyle and J. Tomiyama, Bounded topological orbit equivalence and $C^{*}$-algebras, J. Math. Soc. Japan 50 (1998), no. 2, 317-329.

[49] M. Boyle and J.B. Wagoner, Positive algebraic K-theory and subshifts of finite type, Modern dynamical systems and applications, 45-66, Cambridge Univ. Press, Cambridge, 2004.

[50] O. Bratteli, P. Jorgensen, K.H. Kim and F. Roush, Decidability of the isomorphism problem for stationary AF-algebras and the associated ordered simple dimension groups, Ergodic Theory Dynam. Systems 21 (2001), no. 6, 1625-1655; Corrigendum Ergodic Theory Dynam. Systems22 (2002), no. 2, 633.

[51] G. Budzban, Semigroups and the generalized road coloring problem, Semigroup Forum 69 (2004), no. 2, 201-208.

[52] R. Burton and J. Steif, New results on measures of maximal entropy, Israel J. Math. 89 (1995), no 1-3, 275-300.

[53] J. Buzzi, Subshifts of quasi-finite type, Invent. Math 159 (2005), no. 2, 369-406.

[54] A. Carbone, Cycles of relatively prime length and the road coloring problem, Israel J. Math 123 (2001), 303-316.

[55] T. M. Carlsen, On $C^{*}$-algebras associated with sofic shifts, J. Operator Theory 49 (2003), no. 1, 203-212.

[56] T. M. Carlsen and S. Eilers, Matsumoto K-groups associated to certain shift spaces, Doc. Math. 9 (2004), 639-671 (electronic).

[57] T. M. Carlsen and K. Matsumoto, Some remarks on the $C^{*}$-algebras associated with subshifts, Math. Scand. 95 (2004), no. 1, 145-160.

[58] H. Cohen, A course in computational algebraic number theory, Graduate Texts in Mathematics 138, Third, Corrected Printing (1996), Springer.

[59] T. Ceccherini-Silberstein and M. Coornaert, The Garden of Eden theorem for linear cellular automata, Ergodic Theory Dynam. Systems 26 (2006), no. 1, 53-68.

[60] T. Ceccherini-Silberstein and M. Coornaert, Cellular automata and groups, Encyclopaedia of Complexity and System Science, Springer-Verlag, 2008.

[61] J. Cuntz and W. Krieger, A class of $C^{*}$-algebras and topological Markov chains, Invent. Math. 56 (1980), 251-268.

[62] J. Cuntz and W. Krieger, Topological Markov chains with dicyclic dimension groups, J. Reine Angew. Math. 320 (1980), 44-51.

[63] A. Desai, Subsystem entropy for $\mathbb{Z}^{d}$ sofic shifts, Indagationes Mathematicae 17 (2006), No. 3, 353-360.

[64] T. Downarowicz, Entropy Structure, J. Anal. Math. 96 (2005), 57-116.

[65] T. Downarowicz and S. Newhouse, Symbolic extensions in smooth dynamical systems, Invent. Math 160 (2005), no. 3, 453-499.

[66] T. Downarowicz and J. Serafin, Possible entropy functions, Israel J. Math. 135 (2003), $221-250$.

[67] S. Eilers, $C^{*}$-algebras associated to dynamical systems, Discrete Contin. Dyn. Syst. 15 (2006), no. 1, 177-192.

[68] M. Einsiedler, Invariant subsets and invariant measures for irreducible actions on zerodimensional groups, Bull. London Math. Soc. 36 (2004), no. 3, 321-331.

[69] M. Einsiedler, Isomorphism and measure rigidity for algebraic actions on zerodimensional groups, Monatsh. Math. 144 (2005), no. 1, 39-69.

[70] M. Einsiedler and E. Lindenstrauss, Rigidity properties of $\mathbb{Z}^{d}$-actions on tori and solenoids, Electron. Res. Announc. Amer. Math. Soc. 9 (2003), 99-110 (electronic).

[71] M. Einsiedler and K. Schmidt. The adjoint action of an expansive algebraic $\mathbb{Z}^{d}$ action, Monatsh. Math. 135 (2002), no. 3, 203-220.

[72] M. Einsiedler and S. Tuncel, When does a polynomial ideal contain a positive polynomial?, Effective methods in algebraic geometry (Bath, 2000), J. Pure Appl. Algebra 164 (2001), no. 1-2, 149-152.

[73] M. Einsiedler, R. Mouat and S. Tuncel, When does a submodule of $\left(\mathbb{R}\left[x_{1}, \ldots, x_{k}\right]\right)^{n}$ contain a positive element?, Monatsh. Math. 140 (2003), no. 4, 267-283.

[74] P. Ferrari, A. Maass, S. Martinez and P. Ney, Cesaro mean distribution of group automata starting from measures with summable decay, Ergodic Theory Dynam. Systems 20 (2000), 1657-1670. 
[75] , F. Fiorenzi, Semi-strongly irreducible shifts, Adv. in Appl. Math. 32 (2004), no. 3, 421-438.

[76] D. Fisher and K. Whyte, When is a group action determined by its orbit structure? Geom. Funct. Anal. 13 (2003), no. 6, 1189-1200.

[77] N. Pytheas Fogg, Substitutions in dynamics, Arithmetics and Combinatorics, SpringerVerlag, Berlin, 2002.

[78] N. A. Friedman and D. S. Ornstein, On isomorphism of weak Bernoulli transformations, Advances in Math. 5 (1970), 365-394.

[79] M. Fujiwara, Conjugacy for one-sided sofic systems, Dynamical systems and singular phenomena (Kyoto, 1986), 189-202, World Sci. Adv. Ser. Dynam. Systems 2, World Sci. Publishing, Singapore, 1987.

[80] H. Furstenberg, Disjointness in ergodic theory, minimal sets, and a problem in Diophantine approximation, Math. Systems Theory 1 (1967), 1-49.

[81] T. Giordano, I.F. Putnam and C. F. Skau. Topological orbit equivalence and $C^{*}$-crossed products, J. Reine Angew. Math. 469 (1995), 51-111.

[82] T. Giordano, H. Matui, I.F. Putnam and C. F. Skau, Orbit equivalence for Cantor minimal $\mathbb{Z}^{2}$ systems, preprint (2006).

[83] J. Goldberger, D. Lind and M. Smorodinsky, The entropies of renewal systems, Israel J. Math. 75 (1991), no. 1, 49-64.

[84] R. Gomez, Positive K-theory for finitary isomorphisms of Markov chains, Ergodic Theory Dynam. Systems 23 (2003), no. 5, 1485-1504.

[85] M. Gromov, Endomorphisms of symbolic algebraic varieties, J. Eur. Math. Soc. (JEMS) 1 (1999), no. 2, 109-197.

[86] Young-Hwa Ha, Conjugation and strong shift equivalence, Comm. Korean Math. Soc. 11 (1996), No. 1, 191-199.

[87] T. Hamachi and M. Nasu, Topological conjugacy for 1-block factor maps of subshifts and sofic covers, Proceedings of Maryland Special Year in Dynamics 1986-87, Springer Lecture Notes in Math. 1342 (1988), 251-260, Springer-Verlag.

[88] D. Handelman, Positive polynomials and product type actions of compact groups, Mem. Amer. Math. Soc. 54 (1985), no. 320.

[89] B. Hasselblatt, Problems in dynamical systems and related topics, in Dynamics, Ergodic Theory, and Geometry, MSRI Publications 54 (2007), 273-325.

[90] M. Hochman and T. Meyerovitch, A characterization of the entropies of multidimensional shifts of finite type, Mathematics ArXiv DS/0703206 (2007).

[91] M. Hochman, Recursively enumerable dynamics, cellular automata and subdynamics of SFTs, preliminary manuscript 2007.

[92] C. Hoffman, A Markov random field which is $K$ but not Bernoulli, Israel J. Math. 112 (1999), 249-269.

[93] B. Host, A. Maass and S. Martinez, Uniform Bernoulli measure in dynamics of permutative cellular automata with algebraic local rules, Discrete Contin. Dyn. Syst. 9 (2003), 1423-1446.

[94] A. S. Johnson, Measures on the circle invariant under multiplication by a nonlacunary subsemigroup of the integers, Israel J. Math. 77 (1992), no. 1-2, 211-240.

[95] A. del Junco, Finitary coding of Markov random fields, Z. Wahrsch. Verw. Gebiete 52, 193-202.

[96] J. Kari, Rice's theorem for the limit sets of cellular automata, Theoret. Comput. Sci $\mathbf{1 2 7}$ (1994), 229-254.

[97] J. Kari, Theory of cellular automata: a survey, Theoret. Comput. Sci. 334 (2005), no. $1-3,3-33$.

[98] J. Kari, The tiling problem and undecidability in cellular automata, Encyclopedia of Complexity and System Science, Springer-Verlag, 2008.

[99] S.Katok and I. Ugarcovici, Symbolic dynamics for the modular surface and beyond, Bulletin A.M.S. 44, No. 1 (2007), 87-132.

[100] M. Keane and M. Smorodinsky, Finitary isomorphisms of irreducible Markov shifts, Israel J. Math. 34 (1979), no. 4, 281-286 (1980).

[101] K.H. Kim and F.W. Roush, On strong shift equivalence over a Boolean semiring, Ergodic Theory Dynam. Systems 6 (1986), no. 1, 81-97. 
[102] K.H. Kim and F.W. Roush, Decidability of shift equivalence, Dynamical systems (College Park, MD, 1986-87), 374-424, Lecture Notes in Math. 1342 Springer, Berlin, 1988.

[103] K.H. Kim and F.W. Roush, Full shifts over $R^{+}$and invariant tetrahedra, Pure Math. Appl. Ser. B 1 (1990), no. 4, 251-256 (1991).

[104] K. H. Kim and F.W. Roush, An algorithm for sofic shift equivalence, Ergodic Theory Dynam. Systems 10 (1990), 381-393.

[105] K.H. Kim and F.W. Roush, Path components of matrices and strong shift equivalence over $Q^{+}$, Linear Algebra Appl. 145 (1991), 177-186.

[106] K.H. Kim and F.W. Roush, Strong shift equivalence over subsemirings of $Q^{+}$, Pure Math. Appl. Ser. B 2 (1991), no. 1, 33-42.

[107] K.H. Kim and F.W. Roush, Strong shift equivalence of Boolean and positive rational matrices, Linear Algebra Appl. 161 (1992), 153-164.

[108] K.H. Kim and F.W. Roush, Topological classification of reducible subshifts, Pure Math. Appl. Ser. B 3 (1992), no. 2-4, 87-102 (1993).

[109] K.H. Kim and F.W. Roush, Williams' Conjecture is false for reducible subshifts, J. Amer. Math. Soc. 5 (1992), 213-215.

[110] K.H. Kim and F.W. Roush, Free $Z_{p}$ actions on subshifts Pure Math. Appl. 8 (1997), no. 2-4, 293-322.

[111] K.H. Kim and F.W. Roush, The Williams conjecture is false for irreducible subshifts, Electron. Res. Announc. Amer. Math. Soc. 3 (1997),105-109 (electronic).

[112] K.H. Kim and F.W. Roush, The Williams conjecture is false for irreducible subshifts, Ann. of Math. (2) 149 (1999), no. 2, 545-558.

[113] K.H.Kim, N.Ormes and F.W. Roush, The spectra of nonnegative integer matrices via formal power series, J. Amer. Math. Soc. 13 (2000), 773-806.

[114] K.H. Kim, F.W. Roush and J.B. Wagoner, Automorphisms of the dimension group and gyration numbers of automorphisms of a shift, J. Amer. Math. Soc. 5 (1992), 191-212.

[115] K.H. Kim, F.W. Roush and J.B. Wagoner, Characterization of inert actions on periodic points I, Forum Math. 12 (2000), 565-602.

[116] K.H. Kim, F.W. Roush, and J.B. Wagoner, Characterization of inert actions on periodic points II, Forum Math. 12,671-712.

[117] K.H. Kim, F.W. Roush, and S. G. Williams, Duality and its consequences for ordered cohomology of finite type subshifts, Combinatorial \& computational mathematics (Pohang, 2000), 243-265, World Sci. Publ., River Edge, NJ, 2001.

[118] B. Kitchens. Expansive dynamics on zero-dimensional groups, Ergodic Theory Dynam. Systems 7 (1987), 249-261.

[119] B.P. Kitchens, Symbolic dynamics. One-sided, two-sided and countable state Markov shifts, Springer-Verlag (1998).

[120] B. Kitchens, The structure of d-dimensional vector shifts and convolutional codes, 18 pages, unpublished manuscript (2003).

[121] B. Kitchens, B. Marcus and P. Trow, Eventual factor maps and compositions of closing maps, Ergodic Theory Dynam. Systems 11 (1991), no. 1, 85-113.

[122] W. Krieger, On the subsystems of topological Markov chains, Ergodic Theory Dynam. Systems 2 (1982), no. 2, 195-202.

[123] W. Krieger, On sofic systems I, Israel J. Math. 48 (1984), 305-330.

[124] W. Krieger and K. Matsumoto, A class of invariants of the topological conjugacy of subshifts, Ergodic Theory Dynam. Systems 24 (2004), no. 4, 1155-1171.

[125] P. Kurka, Languages, equicontinuity and attractors in cellular automata, Ergodic Theory Dynam. Systems 17 (1997), 417-433.

[126] P. Kưrka, Topological and symbolic dynamics Cours Spcialiss [Specialized Courses] 11, Socit Mathmatique de France, Paris, 2003.

[127] D. Lind and B. Marcus, An introduction to Symbolic Dynamics and Coding, Cambridge University Press (1995).

[128] D. Lind and K. Schmidt, Symbolic and algebraic dynamical systems, Handbook of dynamical systems, Vol. 1A, 765-812, North-Holland, Amsterdam, 2002.

[129] E.Lindenstrauss, Rigidity of multiparameter actions (survey paper), Israel J. Math. 149 (2005), 199-226.

[130] A. Maass, On the sofic limit sets of cellular automata, Ergodic Theory Dynam. Systems 15 (1995), no. 4, 663-684. 
[131] A. Maass, S. Martinez, M. Pivato and R. Yassawi, Asymptotic randomization of subgroup shifts by linear cellular automata, Ergodic Theory Dynam. Systems 26 (2006), no. 4, 1203-1224.

[132] K. Madden, A single nonexpansive, nonperiodic rational direction, Complex Systems 12 (2000), no. 2, 253-260.

[133] M. Maller and M. Shub, The integral homology of Smale diffeomorphisms, Topology 24 (1985), 153-164.

[134] B. Marcus, Factors and extensions of full shifts, Monatsh. Math. 88 (1979), no. 3, 239247.

[135] B. Marcus, K. Petersen and S. Williams, Transmission rates and factors of Markov chains, Conference in modern analysis and probability (New Haven, Conn., 1982), 279293, Contemp. Math. 26, Amer. Math. Soc., Providence, RI, 1984.

[136] B. Marcus and S. Tuncel, The weight-per-symbol polytope and scaffolds of invariants associated with Markov chains, Ergodic Theory Dynam. Systems 11 (1991), 129-180.

[137] B. Marcus and S. Tuncel, Entropy at a weight-per-symbol and embeddings of Markov chains, Invent. Math. 102 (1990), no. 2, 235-266.

[138] B. Marcus and S. Tuncel, Matrices of polynomials, positivity, and finite equivalence of Markov chains, J. Amer. Math. Soc. 6 (1993), no. 1, 131-147.

[139] K. Matsumoto, On $C^{*}$-algebras associated with subshifts, Internat. J. Math. 8 (1997), 357-374.

[140] K. Matsumoto, Presentations of subshifts and their topological conjugacy invariants, Documenta Math. 4 (1999), 285-340.

[141] K. Matsumoto, Dimension groups for subshifts and simplicity of the associated $C^{*}$ algebras, J. Math. Soc. Japan 51 (1999), no. 3, 679-698.

[142] K. Matsumoto, $C^{*}$-algebras associated with presentations of subshifts, Doc. Math. 7 (2002), 1-30 (electronic).

[143] K. Matsumoto, Topological entropy in $C^{*}$-algebras associated with lambda-graph systems, Ergodic Theory Dynam. Systems 25 (2005), no. 6, 1935-1950.

[144] M. Morse and G. A. Hedlund, Symbolic dynamics II: Sturmian trajectories, Amer. J. Math. 62 (1940), 1-42.

[145] R. Mouat and S. Tuncel, Constructing finitary isomorphisms with finite expected coding times, Israel J. Math. 132 (2002), 359-372.

[146] S. Mozes, Tilings, substitution systems and dynamical systems generated by them, J. Analyse Math. 53 (1989), 139-186.

[147] M. Nasu, Topological conjugacy for sofic systems, Ergodic Theory Dynam. Systems 6 (1986), 265-280

[148] M. Nasu, Topological conjugacy for sofic systems and extensions of automorphisms of finite subsystems of topological Markov shifts, Proceedings of Maryland Special Year in Dynamics 1986-87, Springer Lecture Notes in Math. 1342 (1988), 564-607,SpringerVerlag.

[149] M. Nasu, A Note on Nonexpansive, Essentially LR Automorphisms of Topological Markov Shifts, Research Reports of the Faculty of Engineering, Mie University, Vol.17, Dec. 1992, Tsu, Mie, Japan.

[150] M. Nasu, Textile systems for endomorphisms and automorphisms of the shift, Mem. Amer. Math. Soc. 114 (1995), no. 546.

[151] M.Nasu, Maps in symbolic dynamics, in Lecture Notes of the Tenth KAIST Mathematics Workshop, ed. Geon Ho Choe, Korea Advanced Institute ofScience and Technology Mathematics Research Center, Taejon, Korea.

[152] M. Nasu, The dynamics of expansive invertible onesided cellular automata, Trans. Amer. Math. Soc. 354 (2002), no. 10, 4067-4084

[153] M. Nasu, Nondegenerate q-biresolving textile systems and expansive automorphisms of onesided full shifts, Trans. Amer. Math. Soc. 358 (2006), no. 2, 871-891.

[154] M. Nasu, Textile systems and onesided resolving automorphisms and endomorphisms of the shift, Ergodic Theory Dynam. Systems, to appear.

[155] M. Nivat, invited talk at ICALP, Bologna, 1997.

[156] W. Parry, Finitary isomorphisms with finite expected code lengths, Bull. London Math. Soc. 11 (1979), no. 2, 170-176. 
[157] W. Parry and K. Schmidt. Natural coefficients and invariants for Markov shifts, Inventiones Math. 76 (1984), 15-32.

[158] W. Parry and R.F. Williams, Block coding and a zeta function for Markov chains, Proc. London Math. Soc. 35 (1977), 483-495.

[159] D. Perrin, On positive matrices, Theoret. Comput. Sci. 94 (1992), no. 2, 357-366.

[160] M. Pivato, Invariant measures for bipermutative cellular automata, Discrete Contin. Dyn. Syst. 12 (2005), 723-736.

[161] M. Pivato, The ergodic theory of cellular automata, preliminary manuscript (2007).

[162] M. Pivato and R. Yassawi, Limit measures for affine cellular automata II, Ergodic Theory Dynam. Systems 24 (2004), 1961-1980.

[163] M. Pivato and R. Yassawi, Asymptotic randomization of sofic shifts by linear cellular automata, Ergodic Theory Dynam. Systems 26 (2006), 1177-1201.

[164] M. Pollicott and H. Weiss, Free energy as a dynamical invariant (or Can you hear the shape of a potential?), Comm. Math. Phys. 240 (2003), no. 3, 457-482.

[165] A. Quas and A. Sahin, Entropy gaps and locally maximal entropy in $\digamma^{d}$ subshifts, Ergodic Theory Dynam. Systems 23 (2003), no. 4, 1227-1245.

[166] A. Quas and L. Zamboni, Periodicity and local complexity, Theoret. Comput. Sci. 319 (2004), no. 1-3, 229-240.

[167] M. Quefflec, Substitution dynamical systems - spectral analysis, Lecture Notes in Mathematics 1294, Springer-Verlag, Berlin, 1987.

[168] G. Rauzy, Nombres algbriques et substitutions, Bull. Soc. Math. France 110 (1982), no. 2, 147-178.

[169] R. M. Robinson, Undecidability and nonperiodicity for tilings of the plane, Invent. Math. 12 (1971), 177-209.

[170] M. Rørdam and E. Størmer, Classification of nuclear $C^{*}$-algebras. Entropy in Operator Algebras, Encyclopedia of Math. Sci 126, Operator Algebras and Non-commutative Geometry, 7, 147-198, Springer, 2002.

[171] J. Rosenberg, Algebraic K-theory and its applications, Graduate Texts in Mathematics 147, Springer-Verlag (1994).

[172] D. J. Rudolph, $\times 2$ and $\times 3$ invariant measures and entropy, Ergodic Theory Dynam. Systems 10 (1990), no. 2, 395-406.

[173] M. Sablik, Measure rigidity for algebraic bipermutative cellular automata, Ergodic Theory Dynam. Systems, to appear.

[174] K. Schmidt, On periodic expansions of Pisot numbers and Salem numbers, Bull. London Math. Soc. 12 (1980), no. 4, 269-278.

[175] K. Schmidt, Hyperbolic structure preserving isomorphisms of Markov shifts, Israel J. Math. 55 (1986), no. 2, 213-228.

[176] K. Schmidt, Hyperbolic structure preserving isomorphisms of Markov shifts. II, Israel J. Math. 58 (1987), no. 2, 225-242.

[177] K. Schmidt, Dynamical systems of algebraic origin, Progress in Mathematics 128, Birkhauser Verlag (1995).

[178] K. Schmidt, Algebraic $\mathbb{Z}^{d}$ actions, Lecture notes of PIMS Distinguished Chair Lectures, Pacific Institute for the Mathematical Sciences (2002), 61 pages.

[179] K. Schmidt, Algebraic $\mathbb{Z}^{d}$-actions on zero-dimensional compact abelian groups, Modern dynamical systems and applications, 185-209, Cambridge Univ. Press, Cambridge, 2004.

[180] S. Silberger, Subshifts of the three dot system, Ergodic Theory Dynam. Systems 25 (2005), no. 5, 1673-1687.

[181] M. Sullivan, An invariant of basic sets of Smale flows, Ergodic Theory Dynam. Systems 17 (1997), 1437-1448; Errata, Ergodic Theory Dynam. Systems 18 (1998), 1047.

[182] M. Sullivan, Invariants of twist-wise flow equivalence, Electron. Res. Announc. Amer. Math. Soc. 3 (1997), 126-130 (electronic).

[183] M. Sullivan, Invariants of twist-wise flow equivalence, Discrete Contin. Dynam.Systems 4 (1998), 475-484.

[184] K. Thomsen, On the structure of a sofic shift space, Trans. Amer. Math. Soc. 356 (2004), no. 9, 3557-3619.

[185] K. Thomsen, On the structure of beta shifts, Algebraic and topological dynamics, 321332, Contemp. Math., 385, Amer. Math. Soc., Providence, RI, 2005.

[186] A. N. Trahtman, The road coloring problem, Math Arxiv 0709.0099 preprint (2007). 
[187] S. Tuncel, Faces of Markov chains and matrices of polynomials, in Symbolic dynamics and its applications (New Haven, CT, 1991), 391-422, Contemp. Math. 135, Amer. Math. Soc., Providence, RI, 1992.

[188] S. Tuncel, Coefficient rings for beta function classes of Markov chains, Ergodic Theory Dynam. Systems 20 (2000), 1477-1493.

[189] F. von Haessler, Automatic sequences, de Gruyter Expositions in Mathematics 36, Walter de Gruyter \& Co., Berlin, 2003.

[190] J. B. Wagoner, Strong shift equivalence theory and the shift equivalence problem, Bull. Amer. Math. Soc. (N.S.) 36 (1999), no. 3, 271-296.

[191] B. Weiss, Sofic groups and dynamical systems, Ergodic theory and harmonic analysis (Mumbai, 1999). Sankhyā Ser. A 62 (2000), no. 3, 350-359.

[192] R.F. Williams, Classification of subshifts of finite type, Annals of Math. 98 (1973), 120153; Errata, Annals of Math. 99 (1974), 380-381.

Department of Mathematics, University of Maryland, College Park, MD 20742-4015, U.S.A.
E-mail address: mmb@math.umd.edu
Home page: www.math.umd.edu/ mmb 